\section{BMJ Global Health}

\title{
A systematic review on ethical challenges of 'field' research in low- income and middle-income countries: respect, justice and beneficence for research staff?
}

\author{
Janina Isabel Steinert (D) , ${ }^{1,2}$ David Atika Nyarige, ${ }^{3}$ Milan Jacobi, ${ }^{4}$ Jana Kuhnt, ${ }^{5}$ \\ Lennart Kaplan ${ }^{6,7}$
}

To cite: Steinert Jl,

Atika Nyarige $\mathrm{D}$, Jacobi $\mathrm{M}$, et al. A systematic review on ethical challenges of 'field' research in low-income and middle-income countries: respect, justice and beneficence for research staff?BMJ Global Health 2021;6:e005380. doi:10.1136/ bmjgh-2021-005380

Handling editor Seye Abimbola

- Additional online supplemental material is published online only. To view, please visit the journal online (http://dx.doi.org/10.1136/ bmjgh-2021-005380).

JK and LK are joint senior authors.

Received 15 February 2021 Accepted 24 June 2021

Check for updates

\section{(c) Author(s) (or their} employer(s)) 2021. Re-use permitted under CC BY-NC. No commercial re-use. See rights and permissions. Published by BMJ.

For numbered affiliations see end of article.

\section{Correspondence to} Dr Janina Isabel Steinert; janina.steinert@tum.de

\section{ABSTRACT}

Introduction Primary data collection in low-income and middle-income countries (LMICs) is associated with a range of ethical complexities. Considerations on how to adequately ensure the well-being of research staff are largely neglected in contemporary ethics discourse. This systematic review aims to identify the ethical challenges that research staff across different hierarchical levels and scientific disciplines face when conducting research in LMICs.

Methods We searched 13 electronic databases and handsearched publications in six selected journals as well as the reference lists of all included studies. No restrictions were applied with respect to the publication date, research design, and target population.

Results 23151 studies were retrieved, 183 of which met our inclusion criteria. We identified nine different types of ethical challenges that research staff may be exposed to during field research, including (1) role conflicts that can emerge from participants' help requests and the high level of deprivation found in certain study settings, (2) feelings of guilt and (3) detrimental mental health impacts. Further challenges were (4) sexual harassment (5) safety risks and (6) political repression, particularly in postconflict, disaster-ridden or autocratic study contexts. Additionally, studies reported (7) inadequate working conditions and (8) power imbalances within research teams, while (9) ethics boards were found to be ill equipped to anticipate and address emerging risks, thus increasing the ethical liability of researchers.

Conclusion This review revealed several complex ethical challenges that research staff may face during data collection. In order to achieve the Sustainable Development Goal 8.8 on 'safe and secure working environments' and to protect research staff from harm, amendments must urgently be made to current ethical standards. PROSPERO registration number CRD42019131013

\section{INTRODUCTION}

The importance of research ethics was tragically recognised in the wake of World War II

\section{Key questions}

What is already known?

- Six existing non-systematic reviews discuss selected ethical challenges that affect research staff during data collection endeavours in low-income and middle-income countries (LMICS).

- None of the existing reviews presented a comprehensive overview of ethical challenges or outlined safeguarding mechanisms for research staff.

What are the new findings?

- This is the first systematic review to date that sheds light on the various ethical challenges presented to research staff during data collection in LMICs.

- Data collection in LMICs can carry harmful consequences for the involved research staff, including threats to their physical and emotional well-being and intricate role conflicts.

- Further challenges comprise precarious employment conditions, inadequate remuneration, intellectual exploitation and feelings of guilt and shame that could arise due to vast socioeconomic disparities between researchers and the study population.

What do the new findings imply?

- Formalised ethical guidelines for the adequate protection of research staff as well as institutionalised support structures for data collection projects in LMICs are urgently needed.

- Individual scholars, academic institutions and funding bodies implementing research projects in LMICs need to ensure the integrity and well-being of research staff alongside that of study participants.

and in light of the inhumane medical experimentations undertaken in Germany during the Nazi era. ${ }^{1}$ In consequence, the Nuremberg Code (1947) and later the World Medical Association's Declarations of Geneva (1948) and of Helsinki (1964) were developed to form the ethical cornerstones of biomedical 
research. ${ }^{2}$ In subsequent years, research ethics gained in importance in other scientific disciplines, fuelled by criticism directed at morally reprehensible research studies such as the Tuskegee Syphilis study, ${ }^{3}$ the Stanford-Prison experiment ${ }^{4}$ and the Tearoom Trade study. ${ }^{56}$ In reaction to these studies, the Belmont Report was drafted in 1978, outlining the three fundamental principles of respect, justice and beneficence that guide human subject research until today. ${ }^{7}$

Up to now, the application of these principles has predominantly centred around protecting the well-being of study participants. This includes critical reflections on procedures of informed consent, anonymity and confidentiality concerns, and on the potential exploitation or coercion of study participants. ${ }^{8-10}$ In contrast, ensuring the protection of research staff has played a less dominant role in contemporary ethical discourse, ${ }^{11}$ even though their well-being is highly relevant for the ethical integrity of a project as a whole and for their interactions with research participants in particular. ${ }^{12}$

A specific variant of human subject research involves collection of primary qualitative or quantitative dataoften referred to as 'field research'-in low-income and middle-income countries (LMICs). Large-scale data generation, including empirical data from LMICs, has gained prominence in the past decades spurred by the 'evidence revolution' that cuts across disciplines and that is linked to the rise of impact evaluations. ${ }^{13}{ }^{14}$ While the quest for more evidence may be justified by the ultimate goal to improve the living conditions of research subjects, the required data collection in LMICs often takes place in settings marked by extreme poverty, political instability and highly vulnerable study participants. ${ }^{15} 16$ The ethical complexities of research projects are likely more pronounced in these contexts. However, systematic knowledge on the ethical challenges that are experienced by research staff and on how these are shaped by local contextual factors and institutional frames is currently lacking. ${ }^{11}$

In light of this gap in existing research, this systematic review aims to identify the range of ethical challenges that research staff faces when conducting research in LMICs. The review is broad in scope, capturing the experiences of research staff at different hierarchical levels and assessing research projects implemented across disciplines and world regions.

\section{METHODS}

\section{Search strategy and selection criteria}

We conducted a systematic review in line with the Preferred Reporting Items for Systematic Reviews and Meta-Analyses guidelines and a preregistered protocol to identify reports and studies of ethical challenges that research staff may encounter when conducting research projects. We did not apply any restrictions to the research design, disciplinary focus and target population of identified research projects. We excluded studies that solely focused on ethical challenges pertaining to research participants.

We searched Web of Science, PsycINFO, Scopus, Global Health, EconLit, WHO Regional Office for Africa Library, 3ie Impact Evaluation Repository, DFID's Research4Development, USAID Development Experience Clearing House, African Development Bank Evaluation Reports, Joint Libraries of World Bank and IMF (JOLIS), British Library of Development Studies and the Institute of Development Studies' ELDIS in September 2019. We further handsearched the Journal of Development Studies, Journal of Development Economics, Journal of Development Effectiveness, Journal of Peace Research, BMJ Global Health and Lancet Global Health and checked the reference lists of all included papers for additional relevant works.

We used English search terms only and set up the search string to reflect a broad range of anticipated ethical challenges. We further applied the Campbell Collaboration's LMICs filter to restrict the scope to research projects carried out in LMICs. No restrictions were applied with regards to publication dates. The search string is provided in online supplemental table S1.

After eliminating duplicates, titles and abstracts were screened by DAN, JS, LK and JK. A subset of $10 \%$ of the identified records was double-screened and inter-rater reliability exceeded $90 \%$. In addition, all records that were marked as 'unclear' by one reviewer were triple screened and any disagreements were resolved through group consensus.

\section{Data analysis}

Data from included studies were entered into a piloted data extraction form to capture relevant information from included full text studies on (1) disciplinary focus/research field, (2) setting and context of the field research project, (3) applied methodology (eg, empirical vs theoretical), (4) ethical challenges reported and (5) category of research personnel affected by these ethical challenges. We categorised research staff by hierarchical position (ie, principal investigators (PIs), PhD students, research assistants (RAs) and data collectors) and by their country of origin, thus differentiating between research staff native to the study setting (hereafter referred to as 'local') and research staff from a foreign country (hereafter referred to as 'non-local').

We used thematic analysis to identify nine broad categories of ethical challenges. Thematic analysis was carried out independently by each author, subsequently reviewed by another review author, and flagged ambiguities were resolved by full group consent. Conducting a quantitative meta-analysis was not deemed appropriate, given that the large majority of included studies were based on qualitative and ethnographic work. In addition, given the considerable heterogeneity found with regard to the methodology of included studies, we did not carry out a formal risk of bias assessment. 


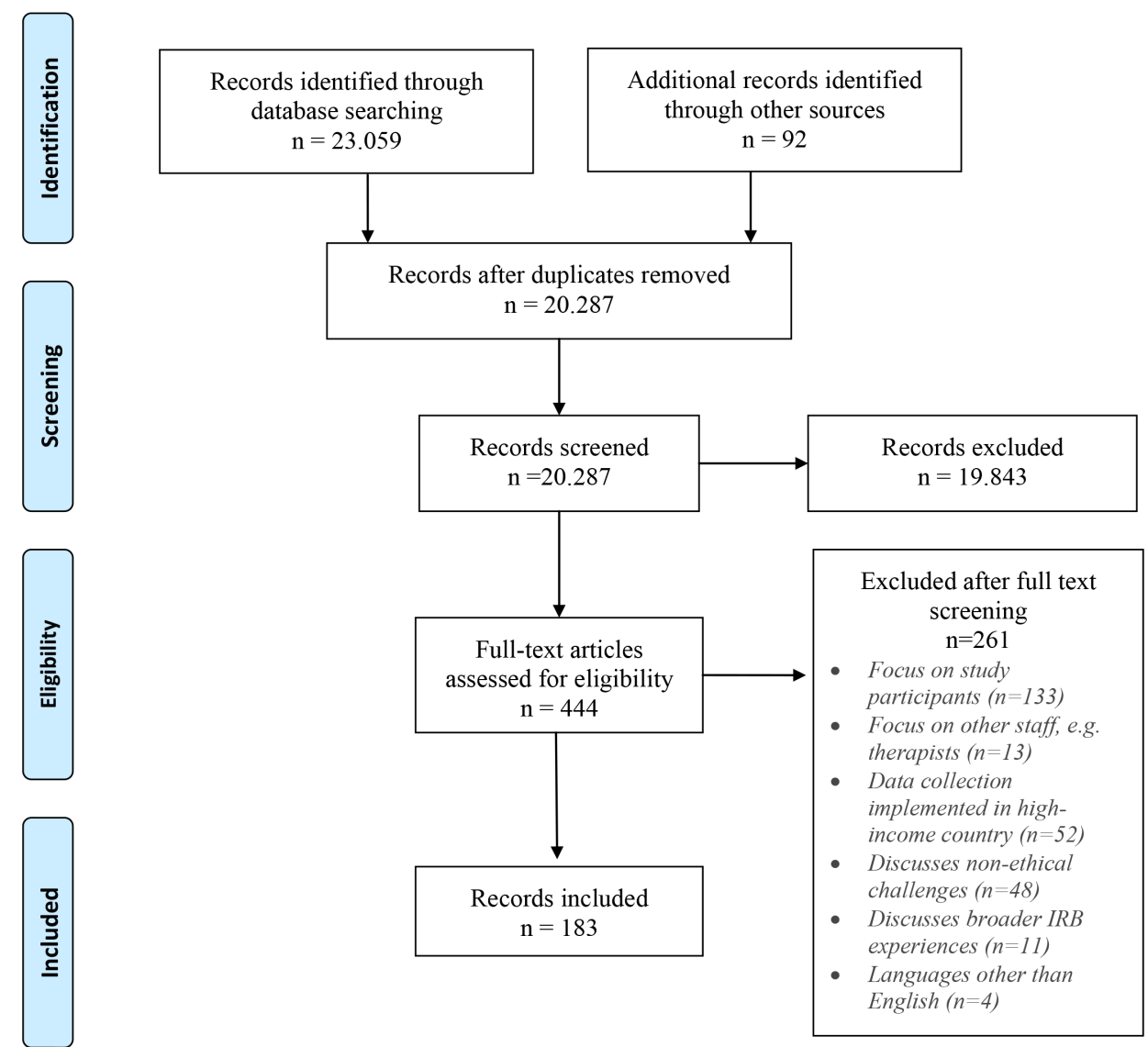

Figure 1 Study selection. IRB, institutional review boards.

\section{Patient and public involvement}

We conducted formative discussions with researchers who were involved in primary data collection in LMICs and represented diverse disciplines, hierarchies and nationalities. In these conversations, the frequency of ethical challenges occurring during 'field' research was reiterated and the urgent need for standardised guidelines on how to protect research staff was emphasised. We intend to present and disseminate our findings to ethics boards, research institutes (eg, Innovation for Poverty Action), and research teams who collect data in LMICs.

\section{RESULTS}

Our database search returned 23059 records and we retrieved 92 additional studies from other sources. We assessed 444 full-text articles, of which 183 met our inclusion criteria (figure 1).

Included studies were implemented across 61 LMICs. Forty-five per cent of the studies were conducted in Africa, 25\% in Asia, 10\% in Latin and South America, 6\% in the Middle East and 4\% were carried out in Europe. Meanwhile, the remaining studies adopted a more global perspective, reporting on challenges to data collection in general (figure 2).

Of 183, $19(10 \%)$ included studies presented a purely normative and theoretical analysis of ethical challenges, while all other studies were based on empirical data and reports. More specifically, 119/183 (65\%) studies were based on autoethnographic field reports, 16/183 (9\%) studies analysed data captured in field journals, 24/183 (13\%) studies included data from qualitative interviews, 6/183 (3\%) studies reported on results from focus group discussions, and only 4/183 (2\%) studies employed quantitative surveys. Ethically challenging situations affected research staff at different hierarchical levels: the majority of studies addressed those affecting PIs $(95 / 183,52 \%$, whereas only $15 / 183,8 \%$ involved local PIs), 27/183 $(15 \%)$ studies focused on non-local $\mathrm{PhD}$ students and 13/183 (7\%) on local PhD students. Of 183, 22 (12\%) studies discussed challenges faced by local data collectors and 25/183 (14\%) highlighted issues for other local research staff. In terms of scientific discipline, 56/183 $(31 \%)$ studies were published in health science, $29 / 183$ $(16 \%)$ in political science (including conflict studies), $17 / 183(9 \%)$ in development studies/development 


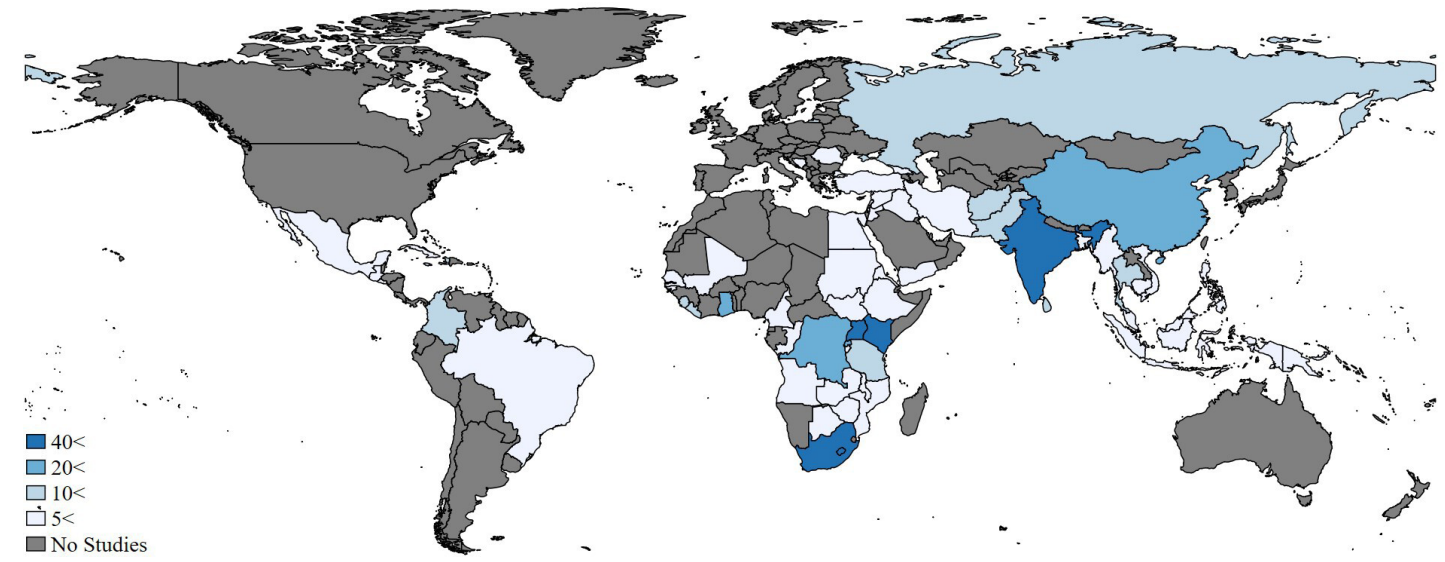

Figure 2 Geographical distribution of included studies.

economics, while the remaining papers were published in other disciplines or in explicitly interdisciplinary social science journals (see table 1 ).

\section{Role conflicts}

The most frequently cited challenge related to role conflicts, cited in 95/183 (52\%) studies (see figure 3). Several included studies, particularly from anthropological and ethnological disciplines, presented detailed reflections on researchers' positionality and on the difficult trade-offs between feeling empathy for fellow human beings and exhibiting the level of detachment appropriate in their positions as objective researchers. ${ }^{1718}$ When data collection is carried out in highly deprived settings, research participants may seek support from research staff. ${ }^{19-22}$ Here, included studies pointed to cases of participants asking for food, ${ }^{23-25}$ medical treatment, ${ }^{26}{ }^{27}$ financial support ${ }^{2528} 29$ or simply seeking to establish a form of friendship with the research staff. ${ }^{26} 2830$ Two studies discussed occasions in which non-local researchers were beseeched to adopt participants' children. ${ }^{31}{ }^{32}$ In light of this, research staff may feel a moral obligation to take on additional roles beyond the scope of their research, for instance by essentially acting in the role of social or aid workers, advocates or counsellors. ${ }^{26}{ }^{32-35}$ This mechanism is likely amplified when the study targets vulnerable populations such as orphans or survivors of natural disasters, wars and violence. ${ }^{36}{ }^{37}$ Participatory research forms may be most prone to provoking such role conflicts due to the greater emotional proximity between participants and researchers. The perceived need for researchers to take on additional responsibilities beyond their immediate research project is also likely increased in settings marked by heavily under-resourced and understaffed service infrastructure, in which material and emotional support and help can thus not be effectively referred on to external professionals. ${ }^{38}$ More importantly, if in the process of a research study, participants disclose information that flags them as being at acute, life-threatening risk, research staff may be faced with a highly complex ethical trade-off between violating the principle of participant confidentiality on the one hand and a moral obligation to protect them from harm on the other hand. ${ }^{39-41}$ Role and positionality conflicts often continue to exist beyond the duration of the project, when research staff members feel obliged to keep providing some form of support to participants, who in turn continue to reach out to them. $^{3042}$

\section{Feelings of guilt}

In contrast to the externally projected roles discussed above, 40/183 (22\%) studies noted that research staff can also internalise feelings of guilt and cast doubts on the legitimacy of the research project as a whole. Related to the difficulties cited above with regard to spelling out clearly defined boundaries for the roles and responsibilities taken on by researchers, data collection can call into question fundamental considerations of reciprocity and responsibility. ${ }^{40} 4344$ That is, while research participants invest their time to take part in the study and disclose detailed information during interviews, researchers may feel unable to substantially improve participants' lives. ${ }^{45-48}$ Included studies presented narratives on how the inability to 'give something back' to participants can evoke feelings of 'tremendous guilt', ${ }^{49}$ 'helplessness', ${ }^{46}$ and that members of the research staff may 'question the purpose of (their) work as a researcher'. ${ }^{50}$ Guilt and shame are more likely internalised if large structural inequalities exist between research participants and researchers. These dynamics were particularly pronounced in studies carried out in disaster and humanitarian contexts. ${ }^{185152}$ Some studies also question the legitimacy of Western researchers who 'speak for the third world', ${ }^{53}$ which directly ties into the aspect of power dynamics discussed below.

\section{Emotional distress and poor mental health}

Another ethical challenge that 50/183 (27\%) included studies referred to was emotional distress. This predominantly occurred in research projects implemented in disaster settings or fragile and conflict-affected countries, and in projects targeting survivors of sexual and physical violence. For instance, in one included study, carried out in a camp for displaced persons, research staff witnessed 
Table 1 Characteristics of included studies

\begin{tabular}{|c|c|c|c|c|c|}
\hline Study reference & Scientific discipline & $\begin{array}{l}\text { Country of } \\
\text { study }\end{array}$ & $\begin{array}{l}\text { Subjects of ethical } \\
\text { assessment }\end{array}$ & Methodological approach & Ethical challenges \\
\hline $\begin{array}{l}\text { Abimanyi-Ochom } \\
\text { (2017) }\end{array}$ & Heath Sciences & Uganda & $\begin{array}{l}\text { PhD (non-local/ } \\
\text { local), RAsRAs (gate } \\
\text { keepers...) (local), Data } \\
\text { Collectors }\end{array}$ & Field Journal/Field Notes & $\begin{array}{l}\text { Political repression/ } \\
\text { persecution, Threat to } \\
\text { physical well-being/safety }\end{array}$ \\
\hline Alderman et al (2013) & Development Studies & Various & PI (non-local) & Autoethnographic & Role conflict/Positionality \\
\hline Augustin $(2018)^{45}$ & Political Science & Yemen & $\mathrm{PhD}$ (non-local/local) & Autoethnographic & $\begin{array}{l}\text { Threat to physical well- } \\
\text { being/safety, Doubts about } \\
\text { purpose/legitimacy of } \\
\text { research/guilt }\end{array}$ \\
\hline Azungha (2019) & $\begin{array}{l}\text { Business/Management } \\
\text { Studies }\end{array}$ & Ghana & $\mathrm{PhD}$ (non-local) & Autoethnographic & $\begin{array}{l}\text { Threat to physical well- } \\
\text { being/safety, Threat to } \\
\text { emotional well-being/mental } \\
\text { health, Poor employment/ } \\
\text { work conditions }\end{array}$ \\
\hline Baaz (2019) & Political Sciences & DRC & PI (non-local) & Autoethnographic & $\begin{array}{l}\text { Sexual harassment/gender } \\
\text { discrimination, Threat to } \\
\text { physical well-being/safety, } \\
\text { Role conflicts }\end{array}$ \\
\hline Bachmann (2011) ${ }^{18}$ & $\begin{array}{l}\text { Geography/Agricultural } \\
\text { Science/Environmental } \\
\text { Science }\end{array}$ & Kenya & PI (non-local) & Autoethnographic & $\begin{array}{l}\text { Role conflicts, Threat to } \\
\text { physical well-being/safety, } \\
\text { Doubts about purpose/ } \\
\text { legitimacy of research/guilt }\end{array}$ \\
\hline Bailey (1988) & Sociology & Global & Not explicitly specified & Theoretical & $\begin{array}{l}\text { Unequal power dynamics, } \\
\text { Limits of IRBs/moral } \\
\text { relativism }\end{array}$ \\
\hline Baird (2009) & Political Science & Colombia & $\mathrm{PhD}$ (non-local) & Autoethnographic & $\begin{array}{l}\text { Threat to physical well- } \\
\text { being/safety }\end{array}$ \\
\hline Baird $^{102}(2018)$ & $\begin{array}{l}\text { Other Social Sciences/ } \\
\text { Interdisciplinary }\end{array}$ & Colombia & $\mathrm{PhD}$ (non-local) & $\begin{array}{l}\text { Autoethnographic, Field Journal/ } \\
\text { Field Notes }\end{array}$ & $\begin{array}{l}\text { Threat to physical well- } \\
\text { being/safety, Threat to } \\
\text { emotional well-being/mental } \\
\text { health, Poor employment/ } \\
\text { work conditions, Doubts } \\
\text { about purpose/legitimacy of } \\
\text { research/guilt }\end{array}$ \\
\hline Bell (2013) & $\begin{array}{l}\text { Other Social Sciences/ } \\
\text { Interdisciplinary }\end{array}$ & Cuba & PI (non-local) & & $\begin{array}{l}\text { Unequal power dynamics, } \\
\text { Poor employment/work } \\
\text { conditions }\end{array}$ \\
\hline Belousov et al $(2007)^{68}$ & $\begin{array}{l}\text { Other Social Sciences/ } \\
\text { Interdisciplinary }\end{array}$ & Russia & PI (non-local) & Autoethnographic & $\begin{array}{l}\text { Threat to physical well- } \\
\text { being/safety, Political } \\
\text { repression/persecution }\end{array}$ \\
\hline Belur $(2014)^{17}$ & $\begin{array}{l}\text { Other Social Sciences/ } \\
\text { Interdisciplinary }\end{array}$ & India & PI (non-local) & Autoethnographic & $\begin{array}{l}\text { Threat to physical well- } \\
\text { being/safety, Role conflicts, } \\
\text { Sexual harassment/gender } \\
\text { discrimination }\end{array}$ \\
\hline Berghs $(2010)^{43}$ & Heath Sciences & Sierra Leone & PI (non-local) & Autoethnographic & $\begin{array}{l}\text { Threat to emotional well- } \\
\text { being/mental health, Doubts } \\
\text { about purpose/legitimacy of } \\
\text { research/guilt }\end{array}$ \\
\hline Bonsu et al (2017) & Heath Sciences & Botswana & $\begin{array}{l}\text { PI (non-local), RAs } \\
\text { (non-local), RAs (gate } \\
\text { keepers...) (local) }\end{array}$ & Autoethnographic & $\begin{array}{l}\text { Poor employment/work } \\
\text { conditions }\end{array}$ \\
\hline Bradfield (2012) & Heath Sciences & Global & PI (non-local) & Autoethnographic & $\begin{array}{l}\text { Role conflicts, Unequal } \\
\text { power dynamics }\end{array}$ \\
\hline Brewis (2014) & $\begin{array}{l}\text { Business/Management } \\
\text { Studies }\end{array}$ & & PI (non-local) & Autoethnographic & $\begin{array}{l}\text { Doubts about purpose/ } \\
\text { legitimacy of research/guilt }\end{array}$ \\
\hline $\begin{array}{l}\text { Broad and Reyes } \\
\text { (2008) }\end{array}$ & $\begin{array}{l}\text { Other Social Sciences/ } \\
\text { Interdisciplinary }\end{array}$ & Colombia & PI (non-local) & Autoethnographic & $\begin{array}{l}\text { Doubts about purpose/ } \\
\text { legitimacy of research/guilt, } \\
\text { Threat to physical well- } \\
\text { being/safety }\end{array}$ \\
\hline $\begin{array}{l}\text { Browne and Moffett } \\
(2014)^{42}\end{array}$ & Political Sciences & $\begin{array}{l}\text { Uganda, } \\
\text { Palestine }\end{array}$ & $\mathrm{PhD}$ (non-local) & Autoethnographic & $\begin{array}{l}\text { Threat to emotional well- } \\
\text { being/mental health, Threat } \\
\text { to physical well-being/ } \\
\text { safety, Role conflicts }\end{array}$ \\
\hline Burke et al (2019) & Health Sciences & Senegal & $\begin{array}{l}\text { RAs (gate keepers...) } \\
\text { (local) }\end{array}$ & Autoethnographic & $\begin{array}{l}\text { Threat to emotional well- } \\
\text { being/mental health }\end{array}$ \\
\hline
\end{tabular}


BMJ Global Health

Table 1 Continued

\begin{tabular}{lllll}
\hline Study reference & Scientific discipline & $\begin{array}{l}\text { Country of } \\
\text { study }\end{array}$ & $\begin{array}{l}\text { Subjects of ethical } \\
\text { assessment }\end{array}$ & Methodological approach \\
\hline Calgaro $(2015)^{46}$ & $\begin{array}{l}\text { Other Social Sciences/ } \\
\text { Interdisciplinary }\end{array}$ & Thailand & PhD (non-local) & Autoethnographic
\end{tabular}

Ethical challenges

Threat to emotional wellbeing/mental health, Doubts about purpose/legitimacy of research/guilt

\begin{tabular}{|c|c|c|c|c|c|}
\hline Campbell (2017) & Political Science & Various & PI (non-local) & Normative/Theoretical & $\begin{array}{l}\text { Threat to emotional well- } \\
\text { being/mental health, Threat } \\
\text { to physical well-being/ } \\
\text { safety, Role conflicts, Limits } \\
\text { of IRBs/moral relativism, } \\
\text { Political repression/ } \\
\text { persecution, Doubts about } \\
\text { purpose/legitimacy of } \\
\text { research/guilt }\end{array}$ \\
\hline Canavati et al $(2017)^{26}$ & Heath Sciences & Cambodia & Data Collectors & Field Journal/Field Notes & $\begin{array}{l}\text { Threat to physical well- } \\
\text { being/safety, Poor } \\
\text { employment/work } \\
\text { conditions, Role conflicts }\end{array}$ \\
\hline Caretta (2015) & $\begin{array}{l}\text { Other Social Sciences/ } \\
\text { Interdisciplinary }\end{array}$ & Kenya, Tanzania & PI (non-local) & Field Journal/Field Notes & $\begin{array}{l}\text { Unequal power dynamics, } \\
\text { Sexual harassment/gender } \\
\text { discrimination, Role conflicts }\end{array}$ \\
\hline Casale et al (2011) & Health Sciences & South Africa & Not explicitly specified & Field Journal/Field Notes & Role conflicts \\
\hline Castillo $(2015)^{67}$ & Anthropology/Ethnology & Philippines & PI (non-local) & Autoethnographic & $\begin{array}{l}\text { Threat to physical well- } \\
\text { being/safety, Threat to } \\
\text { emotional well-being/mental } \\
\text { health }\end{array}$ \\
\hline Chaitin $(2003)^{70}$ & Heath Sciences & Israel, Palestine & Not explicitly specified & Autoethnographic & $\begin{array}{l}\text { Threat to physical well- } \\
\text { being/safety }\end{array}$ \\
\hline Chiumento et al (2018) & $\begin{array}{l}\text { Other Social Sciences/ } \\
\text { Interdisciplinary }\end{array}$ & South Asia & PI (non-local) & Autoethnographic & $\begin{array}{l}\text { Poor employment/work } \\
\text { conditions, Doubts about } \\
\text { purpose/legitimacy of } \\
\text { research/guilt }\end{array}$ \\
\hline Coles et al $(2014)^{44}$ & $\begin{array}{l}\text { Other Social Science/ } \\
\text { Interdisciplinary }\end{array}$ & $\begin{array}{l}\text { South Africa, } \\
\text { South America }\end{array}$ & PI (non-local) & Qualitative Interview & $\begin{array}{l}\text { Doubts about purpose/ } \\
\text { legitimacy of research/guilt, } \\
\text { Threat to emotional well- } \\
\text { being/mental health, Threat } \\
\text { to physical well-being/ } \\
\text { safety, Role conflicts }\end{array}$ \\
\hline Crabtree (2019) & Development Studies & $\begin{array}{l}\text { Papua New } \\
\text { Guinea }\end{array}$ & PI (non-local) & Autoethnographic & Role conflicts \\
\hline $\begin{array}{l}\text { Cronin-Furman and } \\
\text { Lake }(2018)^{15}\end{array}$ & $\begin{array}{l}\text { Other Social Sciences/ } \\
\text { Interdisciplinary }\end{array}$ & Various & $\begin{array}{l}\text { PI (non-local), RAs (gate } \\
\text { keepers...) (local), Data } \\
\text { Collectors }\end{array}$ & Autoethnographic & $\begin{array}{l}\text { Threat to physical well- } \\
\text { being/safety, Poor } \\
\text { employment/work } \\
\text { conditions, Unequal power } \\
\text { dynamics }\end{array}$ \\
\hline Davison et al (2013) & Heath Sciences & Jamaica & $\begin{array}{l}\text { RAs (gate keepers...) } \\
\text { (local) }\end{array}$ & Field Journal/Field Notes & $\begin{array}{l}\text { Role conflicts, Doubts } \\
\text { about purpose/legitimacy of } \\
\text { research/guilt }\end{array}$ \\
\hline Dayal et al (2018) & Heath Sciences & India & Not explicitly specified & Literature Review & $\begin{array}{l}\text { Threat to physical well- } \\
\text { being/safety, Threat to } \\
\text { emotional well-being/mental } \\
\text { health }\end{array}$ \\
\hline $\begin{array}{l}\text { Derry and Baum } \\
(1994)^{58}\end{array}$ & Psychology & Various & RAs (non-local) & Other & $\begin{array}{l}\text { Role conflicts, Threat to } \\
\text { emotional well-being/mental } \\
\text { health, Sexual harassment/ } \\
\text { gender discrimination }\end{array}$ \\
\hline Dixit (2012) & Political Sciences & Various & PhD (local) & Autoethnographic & Role conflicts \\
\hline Dodsworth $(2019)^{72}$ & Political Science & various & Not explicitly specified & & Unequal power dynamics \\
\hline Doherty et al (2018) & Health Sciences & Global & PI (non-local) & Theoretical & $\begin{array}{l}\text { Unequal power dynamics, } \\
\text { Role conflicts }\end{array}$ \\
\hline $\begin{array}{l}\text { Dominey-Howes } \\
(2015)^{47}\end{array}$ & $\begin{array}{l}\text { Other Social Sciences/ } \\
\text { Interdisciplinary }\end{array}$ & Various & $\begin{array}{l}\text { PI (non-local), PhD (non- } \\
\text { local) }\end{array}$ & Autoethnographic & $\begin{array}{l}\text { Threat to emotional well- } \\
\text { being/mental health, Role } \\
\text { conflicts, Doubts about } \\
\text { purpose/legitimacy of } \\
\text { research/guilt, Unequal } \\
\text { power dynamics }\end{array}$ \\
\hline D'Souza et al (2018) & Heath Sciences & Jamaica & PI (non-local), PI (local) & Autoethnographic & Role conflicts \\
\hline
\end{tabular}


Table 1 Continued

\begin{tabular}{|c|c|c|c|c|c|}
\hline Study reference & Scientific discipline & $\begin{array}{l}\text { Country of } \\
\text { study }\end{array}$ & $\begin{array}{l}\text { Subjects of ethical } \\
\text { assessment }\end{array}$ & Methodological approach & Ethical challenges \\
\hline Einarsdóttir (2006) & Anthropology/Ethnology & Guinea Bissau & PI (non-local) & Autoethnographic & Role conflicts \\
\hline $\begin{array}{l}\text { Ellsberg and Potts } \\
\text { (2018) }\end{array}$ & Health Sciences & Global & $\begin{array}{l}\text { RAs (gate keepers...) } \\
\text { (local) }\end{array}$ & Review of guidelines & $\begin{array}{l}\text { Threat to physical well- } \\
\text { being/safety, Threat to } \\
\text { emotional well-being/mental } \\
\text { health }\end{array}$ \\
\hline Fidalgo et al (2017) & Heath Sciences & Brazil & Data Collectors & Autoethnographic & $\begin{array}{l}\text { Poor employment/work } \\
\text { conditions }\end{array}$ \\
\hline $\begin{array}{l}\text { Francis and Hemson } \\
\text { (2009) }\end{array}$ & Heath Sciences & South Africa & Data Collectors & Focus group & $\begin{array}{l}\text { Role conflicts, Poor } \\
\text { employment/work } \\
\text { conditions, Threat to } \\
\text { emotional well-being/mental } \\
\text { health }\end{array}$ \\
\hline Fuchs et al (2019) & $\begin{array}{l}\text { Business/Management } \\
\text { Studies }\end{array}$ & China & PhD (non-local) & $\begin{array}{l}\text { Qualitative and quantitative } \\
\text { document/law review }\end{array}$ & $\begin{array}{l}\text { Threat to physical well- } \\
\text { being/safety, Political } \\
\text { repression/persecution }\end{array}$ \\
\hline George (2015) & Political Science & India & PhD (non-local) & Autoethnographic & Role conflicts \\
\hline Gerharz $(2017)^{34}$ & Sociology & Sri Lanka & PI (non-local) & Autoethnographic & $\begin{array}{l}\text { Role conflicts, Threat to } \\
\text { physical well-being/safety, } \\
\text { Poor employment/work } \\
\text { conditions }\end{array}$ \\
\hline $\begin{array}{l}\text { Gheondea-Eladi } \\
(2017)^{40}\end{array}$ & Health Sciences & Romania & PI (local) & Autoethnographic & $\begin{array}{l}\text { Limits of IRBs/moral } \\
\text { relativism, Doubts about } \\
\text { purpose/legitimacy of } \\
\text { research/guilt, Poor } \\
\text { employment/work } \\
\text { conditions, Role conflicts }\end{array}$ \\
\hline Gilmore (2019) & Health Sciences & $\begin{array}{l}\text { Tanzania, } \\
\text { Uganda, Kenya }\end{array}$ & Not explicitly specified & Autoethnographic & $\begin{array}{l}\text { Unequal power dynamics, } \\
\text { Sexual harassment/gender } \\
\text { discrimination }\end{array}$ \\
\hline Girei (2017) & $\begin{array}{l}\text { Business/Management } \\
\text { Studies }\end{array}$ & Uganda & $\mathrm{PI}$ (non-local) & Autoethnographic & $\begin{array}{l}\text { Role conflicts, Unequal } \\
\text { power dynamics }\end{array}$ \\
\hline Glasius et al (2018) & Political Science & Various & $\begin{array}{l}\mathrm{PI} \text { (non-local), PI (local), } \\
\text { PhD (non-local), PhD } \\
\text { (local) }\end{array}$ & Autoethnographic & $\begin{array}{l}\text { Political repression/ } \\
\text { persecution, Threat to } \\
\text { physical well-being/safety, } \\
\text { Doubts about purpose/ } \\
\text { legitimacy of research/guilt, } \\
\text { Threat to emotional well- } \\
\text { being/mental health }\end{array}$ \\
\hline $\begin{array}{l}\text { Glennerster and } \\
\text { Powers (2013) }\end{array}$ & Development Studies & Global & Not explicitly specified & Theoretical & $\begin{array}{l}\text { Role conflicts, Doubts } \\
\text { about purpose/legitimacy of } \\
\text { research/guilt }\end{array}$ \\
\hline Gokah $(2006)^{57}$ & $\begin{array}{l}\text { Other Social Sciences/ } \\
\text { Interdisciplinary }\end{array}$ & $\begin{array}{l}\text { Ghana, Uganda, } \\
\text { Ethiopia, South } \\
\text { Africa }\end{array}$ & PhD (non-local) & Autoethnographic & $\begin{array}{l}\text { Threat to physical well- } \\
\text { being/safety, Threat to } \\
\text { emotional well-being/mental } \\
\text { health, Role conflicts }\end{array}$ \\
\hline Goodhand (2000) & Political Science & $\begin{array}{l}\text { Afghanistan, Sri } \\
\text { Lanka, Liberia }\end{array}$ & PI (non-local) & Autoethnographic & $\begin{array}{l}\text { Threat to physical well- } \\
\text { being/safety, Role conflicts }\end{array}$ \\
\hline Gosh (2018) & $\begin{array}{l}\text { Geography/Agricultural } \\
\text { Science/Environmental } \\
\text { Science }\end{array}$ & India & $\mathrm{PI}$ (non-local) & Autoethnographic & $\begin{array}{l}\text { Threat to physical well- } \\
\text { being/safety }\end{array}$ \\
\hline Gouda et al (2016) ${ }^{63}$ & Heath Sciences & $\begin{array}{l}\text { Papua New } \\
\text { Guinea }\end{array}$ & Data Collectors & $\begin{array}{l}\text { Qualitative Interview, Focus } \\
\text { group }\end{array}$ & $\begin{array}{l}\text { Threat to emotional well- } \\
\text { being/mental health, Role } \\
\text { conflicts, Doubts about } \\
\text { purpose/legitimacy of } \\
\text { research/guilt }\end{array}$ \\
\hline Groes-Green $(2012)^{51}$ & Anthropology/Ethnology & Mozambique & PI (non-local) & Autoethnographic & $\begin{array}{l}\text { Sexual harassment/ } \\
\text { gender discrimination, Role } \\
\text { conflicts, Unequal power } \\
\text { dynamics, Doubts about } \\
\text { purpose/legitimacy of } \\
\text { research/guilt }\end{array}$ \\
\hline Grünenfelder $(2014)^{85}$ & Sociology & Pakistan & Data Collectors & Qualitative Interview & $\begin{array}{l}\text { Sexual harassment/gender } \\
\text { discrimination, Threat to } \\
\text { physical well-being/safety }\end{array}$ \\
\hline Gupta and Kelly (2014) & $\begin{array}{l}\text { Other Social Sciences/ } \\
\text { Interdisciplinary }\end{array}$ & Various & $\mathrm{PI}$ (non-local) & Autoethnographic & $\begin{array}{l}\text { Doubts about purpose/ } \\
\text { legitimacy of research/guilt }\end{array}$ \\
\hline
\end{tabular}


BMJ Global Health

Table 1 Continued

\begin{tabular}{lllll}
\hline Study reference & Scientific discipline & $\begin{array}{l}\text { Country of } \\
\text { study }\end{array}$ & $\begin{array}{l}\text { Subjects of ethical } \\
\text { assessment }\end{array}$ & Methodological approach \\
\hline $\begin{array}{l}\text { Gurol and Wetterich } \\
(2019)\end{array}$ & Political Science & China & PhD (non-local) & Autoethnographic
\end{tabular}

Ethical challenges

\begin{tabular}{|c|c|c|c|c|}
\hline Hamid $(2010)^{19}$ & $\begin{array}{l}\text { Other Social Sciences/ } \\
\text { Interdisciplinary }\end{array}$ & Bangladesh & $\mathrm{PhD}$ (non-local) & Autoethnographic \\
\hline $\begin{array}{l}\text { Hoffman and } \\
\text { Tarawalley (2014) }\end{array}$ & Anthropology/Ethnology & $\begin{array}{l}\text { Liberia, Sierra } \\
\text { Leone }\end{array}$ & PI (non-local), PI (local) & Field Journal/Field No \\
\hline $\mathrm{Hu}(2015)^{65}$ & Political Sciences & China & PI (non-local) & Autoethnographic \\
\hline $\begin{array}{l}\text { Huggins and Glebbeek } \\
\text { (2009) }\end{array}$ & $\begin{array}{l}\text { Other Social Sciences/ } \\
\text { Interdisciplinary }\end{array}$ & Brazil, Guatemala & PI (non-local) & Autoethnographic \\
\hline
\end{tabular}
(2009)

Interdisciplinary

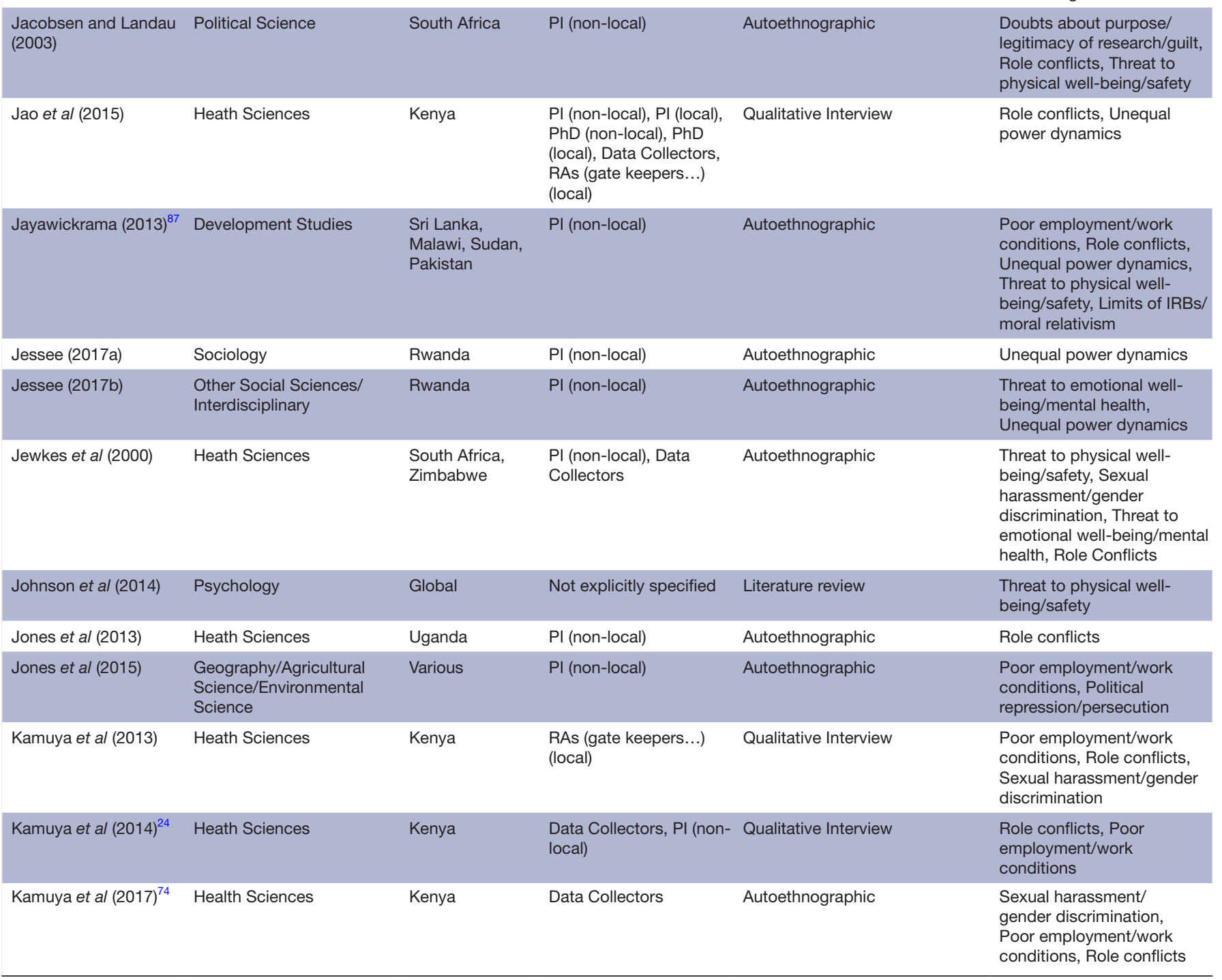


Table 1 Continued

\begin{tabular}{lllll}
\hline Study reference & Scientific discipline & $\begin{array}{l}\text { Country of } \\
\text { study }\end{array}$ & $\begin{array}{l}\text { Subjects of ethical } \\
\text { assessment }\end{array}$ & Methodological approach \\
\hline Kelley et al (2016) & Heath Sciences & $\begin{array}{l}\text { South Africa, } \\
\text { Uganda, Kenya, } \\
\text { Cambodia, } \\
\text { Thailand }\end{array}$ & PI (non-local) & Qualitative Interview \\
& & Role conflicts &
\end{tabular}

Khalid (2014) Development Studies Pakistan PhD (local) Autoethnographic Poor employment/ work conditions, Sexual harassment/gender discrimination, Role conflicts, Limits of IRBs/ moral relativism, Threat to physical well-being/safety

\begin{tabular}{|c|c|c|c|c|c|}
\hline Kingori $(2013)^{23}$ & Heath Sciences & Kenya & Data Collectors & Autoethnographic & $\begin{array}{l}\text { Poor employment/work } \\
\text { conditions, Doubts about } \\
\text { purpose/legitimacy of } \\
\text { research/guilt, Threat to } \\
\text { emotional well-being/mental } \\
\text { health }\end{array}$ \\
\hline $\begin{array}{l}\text { Kingori and Gerrets } \\
(2016)\end{array}$ & Heath Sciences & Various & $\begin{array}{l}\text { PI (non-local), RAs (gate } \\
\text { keepers...) (local) }\end{array}$ & Qualitative Interview & $\begin{array}{l}\text { Role conflicts, Threat to } \\
\text { emotional well-being/mental } \\
\text { health, Poor employment/ } \\
\text { work conditions }\end{array}$ \\
\hline Klocker $(2015)^{56}$ & $\begin{array}{l}\text { Other Social Sciences/ } \\
\text { Interdisciplinary }\end{array}$ & Tanzania & $\begin{array}{l}\text { Data Collectors, PhD } \\
\text { (non-local/local), RAs } \\
\text { (gate keepers...) (local) }\end{array}$ & Qualitative Interview & $\begin{array}{l}\text { Threat to emotional well- } \\
\text { being/mental health }\end{array}$ \\
\hline $\begin{array}{l}\text { Kovats-Bernat } \\
(2002)^{101}\end{array}$ & Anthropology/Ethnology & Haiti & PI (non-local) & Autoethnographic & $\begin{array}{l}\text { Threat to physical well- } \\
\text { being/safety }\end{array}$ \\
\hline Kunnath $(2013)^{53}$ & Anthropology/Ethnology & India & PI (local) & Autoethnographic & $\begin{array}{l}\text { Doubts about purpose/ } \\
\text { legitimacy of research/ } \\
\text { guilt, Political repression/ } \\
\text { persecution, Threat to } \\
\text { emotional well-being/mental } \\
\text { health, Threat to physical } \\
\text { well-being/safety }\end{array}$ \\
\hline Lages et al (2015) & $\begin{array}{l}\text { Business/Management } \\
\text { Studies }\end{array}$ & Global & PI (non-local) & Other & $\begin{array}{l}\text { Poor employment/work } \\
\text { conditions }\end{array}$ \\
\hline $\begin{array}{l}\text { Lake and Parkinson } \\
(2017)\end{array}$ & Political Science & Various & PhD (non-local) & Theoretical & $\begin{array}{l}\text { Threat to physical well- } \\
\text { being/safety }\end{array}$ \\
\hline Lansford et al (2019) & Psychology & Global & Not explicitly specified & Autoethnographic & Unequal power dynamics \\
\hline Lawhon et al (2014) & $\begin{array}{l}\text { Geography/Agricultural } \\
\text { Science/Environmental } \\
\text { Science }\end{array}$ & South Africa & PhD (non-local) & Autoethnographic & $\begin{array}{l}\text { Threat to emotional well- } \\
\text { being/mental health, Limits } \\
\text { of IRBs/moral relativism, } \\
\text { Threat to physical well- } \\
\text { being/safety }\end{array}$ \\
\hline Leach (2006) & Health Sciences & Various & PI (non-local) & Autoethnographic & $\begin{array}{l}\text { Sexual harassment/gender } \\
\text { discrimination }\end{array}$ \\
\hline $\begin{array}{l}\text { Leach and Horne } \\
\text { (2018) }\end{array}$ & Psychology & Russia, Rwanda & PI (non-local) & Focus group & $\begin{array}{l}\text { Doubts about purpose/ } \\
\text { legitimacy of research/guilt, } \\
\text { Role conflicts, Unequal } \\
\text { power dynamics, Limits of } \\
\text { IRBs/moral relativism }\end{array}$ \\
\hline Lombe et al (2012) ${ }^{103}$ & Social Work & Ghana & Not explicitly specified & Autoethnographic & Unequal power dynamics \\
\hline Lund (2012) & $\begin{array}{l}\text { Other Social Sciences/ } \\
\text { Interdisciplinary }\end{array}$ & Sri Lanka & PI (non-local) & Autoethnographic & $\begin{array}{l}\text { Threat to emotional well- } \\
\text { being/mental health, Threat } \\
\text { to physical well-being/ } \\
\text { safety, Doubts about } \\
\text { purpose/legitimacy of } \\
\text { research/guilt, Role conflicts }\end{array}$ \\
\hline Madiega $(2013)^{25}$ & Heath Sciences & Kenya & Data Collectors & Qualitative Interview & $\begin{array}{l}\text { Role conflicts, Poor } \\
\text { employment/work } \\
\text { conditions, Threat to } \\
\text { emotional well-being/mental } \\
\text { health }\end{array}$ \\
\hline
\end{tabular}

Continued 
BMJ Global Health

\begin{tabular}{|c|c|c|c|c|c|}
\hline Study reference & Scientific discipline & $\begin{array}{l}\text { Country of } \\
\text { study }\end{array}$ & $\begin{array}{l}\text { Subjects of ethical } \\
\text { assessment }\end{array}$ & Methodological approach & Ethical challenges \\
\hline $\begin{array}{l}\text { Maier and Monahan } \\
(2009)^{48}\end{array}$ & Psychology & Global & $\begin{array}{l}\text { PI (non-local), PhD (non- } \\
\text { local), RAs (non-local) }\end{array}$ & Qualitative Interview & $\begin{array}{l}\text { Role conflicts, Threat to } \\
\text { physical well-being/safety, } \\
\text { Doubts about purpose/ } \\
\text { legitimacy of research/guilt, } \\
\text { Threat to emotional well- } \\
\text { being/mental health }\end{array}$ \\
\hline Makhoul et al (2014) & Sociology & Lebanon & PI (non-local) & Qualitative Interview & $\begin{array}{l}\text { Unequal power dynamics, } \\
\text { Limits of IRBs/moral } \\
\text { relativism }\end{array}$ \\
\hline Mann (2019) & Political Science & Colombia & PhD (non-local) & Autoethnographic & $\begin{array}{l}\text { Threat to emotional well- } \\
\text { being/mental health, Threat } \\
\text { to physical well-being/ } \\
\text { safety, Sexual harassment/ } \\
\text { gender discrimination, Role } \\
\text { conflicts }\end{array}$ \\
\hline $\begin{array}{l}\text { Mannell and Guta } \\
\text { (2018) }\end{array}$ & Heath Sciences & Rwanda & $\begin{array}{l}\text { RAs (gate keepers...) } \\
\text { (local), PI (non-local) }\end{array}$ & Autoethnographic & Role conflicts \\
\hline Marchais (2020) & $\begin{array}{l}\text { Other Social Sciences/ } \\
\text { Interdisciplinary }\end{array}$ & DRC & $\begin{array}{l}\text { PI (non-local), RAs (non- } \\
\text { local) }\end{array}$ & Autoethnographic & $\begin{array}{l}\text { Unequal power dynamics, } \\
\text { Poor employment/work } \\
\text { conditions, Threat to } \\
\text { physical well-being/safety }\end{array}$ \\
\hline Marchais et al (2020) & Political Science & DRC & Not explicitly specified & Autoethnographic & $\begin{array}{l}\text { Unequal power dynamics, } \\
\text { Role conflicts, Poor } \\
\text { employment/work } \\
\text { conditions, Threat to } \\
\text { physical well-being/safety, } \\
\text { Sexual harassment/gender } \\
\text { discrimination }\end{array}$ \\
\hline Minkler (2004) & Heath Sciences & Global & Not explicitly specified & Theoretical & $\begin{array}{l}\text { Unequal power dynamics, } \\
\text { Poor employment/work } \\
\text { conditions, Role conflicts }\end{array}$ \\
\hline $\begin{array}{l}\text { Molony and Hammett } \\
(2007)^{75}\end{array}$ & $\begin{array}{l}\text { Other Social Sciences/ } \\
\text { Interdisciplinary }\end{array}$ & $\begin{array}{l}\text { South Africa, } \\
\text { Tanzania }\end{array}$ & $\begin{array}{l}\text { PI (non-local), RAs (gate } \\
\text { keepers...) (local) }\end{array}$ & Autoethnographic & $\begin{array}{l}\text { Poor employment/work } \\
\text { conditions, Unequal } \\
\text { power dynamics, Threat } \\
\text { to physical well-being/ } \\
\text { safety, Role conflicts, } \\
\text { Sexual harassment/gender } \\
\text { discrimination }\end{array}$ \\
\hline Molyneux et al (2009) & Development Studies & $\begin{array}{l}\text { Kenya, South } \\
\text { Africa }\end{array}$ & Data Collectors & Autoethnographic & Role conflicts \\
\hline Molyneux et al (2016) & Health Sciences & Kenya & PI (non-local) & $\begin{array}{l}\text { Qualitative Interview, Field } \\
\text { Journal }\end{array}$ & Role conflicts \\
\hline Moncrieffe (2009) & Development Studies & Jamaica & PI (non-local) & Autoethnographic & $\begin{array}{l}\text { Threat to physical well- } \\
\text { being/safety }\end{array}$ \\
\hline Morrell et al (2012) & Sociology & South Africa & $\begin{array}{l}\text { PI (non-local), PhD } \\
\text { (non-local), RAs (gate } \\
\text { keepers...) (local) }\end{array}$ & Autoethnographic & Role conflicts \\
\hline Morris (2015) & $\begin{array}{l}\text { Other Social Sciences/ } \\
\text { Interdisciplinary }\end{array}$ & various & Not explicitly specified & $\begin{array}{l}\text { Quantitative Survey, Qualitative } \\
\text { Interview, Literature Review }\end{array}$ & $\begin{array}{l}\text { Threat to physical well- } \\
\text { being/safety, Poor } \\
\text { employment/work } \\
\text { conditions }\end{array}$ \\
\hline Mosavel $^{54}$ et al (2011) & Heath Sciences & South Africa & $\begin{array}{l}\text { RAs (gate keepers...) } \\
\text { (local) }\end{array}$ & Field Journal & $\begin{array}{l}\text { Poor employment/work } \\
\text { conditions, Threat to } \\
\text { emotional well-being/mental } \\
\text { health, Threat to physical } \\
\text { well-being/safety, Role } \\
\text { conflicts }\end{array}$ \\
\hline Moss et al (2019) & Psychology & $\begin{array}{l}\text { Rwanda, } \\
\text { Zanzibar, Sudan, } \\
\text { Turkey }\end{array}$ & Not explicitly specified & Field Journal & $\begin{array}{l}\text { Threat to physical well- } \\
\text { being/safety, Political } \\
\text { repression/persecution, } \\
\text { Threat to emotional well- } \\
\text { being/mental health }\end{array}$ \\
\hline Motlafi (2018) & Political Sciences & $\begin{array}{l}\text { South Africa, } \\
\text { Rwanda }\end{array}$ & PI (non-local) & Autoethnographic & Unequal power dynamics \\
\hline
\end{tabular}


Table 1 Continued

\begin{tabular}{|c|c|c|c|c|c|}
\hline Study reference & Scientific discipline & $\begin{array}{l}\text { Country of } \\
\text { study }\end{array}$ & $\begin{array}{l}\text { Subjects of ethical } \\
\text { assessment }\end{array}$ & Methodological approach & Ethical challenges \\
\hline Mukherji et al (2014) & $\begin{array}{l}\text { Geography/Agricultural } \\
\text { Science/Environmental } \\
\text { Science }\end{array}$ & Haiti, India & PI (non-local) & Autoethnographic & $\begin{array}{l}\text { Threat to emotional well- } \\
\text { being/mental health, } \\
\text { Role conflicts, Sexual } \\
\text { harassment/gender } \\
\text { discrimination }\end{array}$ \\
\hline
\end{tabular}

Role conflicts, Limits of IRBs/moral relativism, Unequal power dynamics

\begin{tabular}{|llll} 
Muzaila et al (2019) & Political Science & $\begin{array}{l}\text { Republic of the } \\
\text { Congo }\end{array}$ & Not explicitly specified Autoethnographic \\
\hline Mwambari (2019a) & & & \\
\hline Other Social Sciences/ & Uganda & Not explicitly specified Autoethnographic \\
Interdisciplinary
\end{tabular}

Poor employment/work conditions, Unequal power dynamics, Threat to emotional well-being/menta health

Unequal power dynamics, Threat to physical wellbeing/safety, Sexual harassment/gender discrimination, Poor employment/work conditions, Role conflicts, Political repression/ persecution

\begin{tabular}{lllll}
\hline Mwambari (2019b) & $\begin{array}{l}\text { Other Social Sciences/ } \\
\text { Interdisciplinary }\end{array}$ & Various & PI (non-local), PI (local) & Autoethnographic \\
$\begin{array}{l}\text { Mwambari and Owor } \\
(2019)\end{array}$ & Development Studies & Uganda, DRC & PI (non-local), PI (local) & Autoethnographic \\
$\begin{array}{l}\text { Nama and Swartz } \\
(2002)^{21}\end{array}$ & Psychology & South Africa & $\begin{array}{l}\text { RAs (gate keepers...) } \\
\text { (local) }\end{array}$ & Autoethnographic \\
\hline Neto $(2019)^{71}$ & Anthropology/Ethnology & Angola, Zambia & $\begin{array}{l}\text { RAs (gate keepers...) } \\
\text { (local) }\end{array}$ & Autoethnographic
\end{tabular}

Unequal power dynamics, Threat to physical wellbeing/safety

Unequal power dynamics, Poor employment/work conditions

Threat to emotional wellbeing/mental health, Role conflicts

Political repression/ persecution, Threat to physical well-being/ safety, Role conflicts, Poor employment/work conditions

\begin{tabular}{|c|c|c|c|c|c|}
\hline $\begin{array}{l}\text { Nyenyezi et al. (2020) } \\
\text { Editors }\end{array}$ & $\begin{array}{l}\text { Other Social Sciences/ } \\
\text { Interdisciplinary }\end{array}$ & DRC & Data Collectors & Autoethnographic & $\begin{array}{l}\text { Poor employment/work } \\
\text { conditions, Threat to } \\
\text { emotional well-being/mental } \\
\text { health, Unequal power } \\
\text { dynamics, Role conflicts, } \\
\text { Sexual harassment/gender } \\
\text { discrimination, Political } \\
\text { repression/persecution, } \\
\text { Threat to physical well- } \\
\text { being/safety }\end{array}$ \\
\hline O’Mathúna (2009) & Health Sciences & Various & Not explicitly specified & Theoretical & $\begin{array}{l}\text { Threat to physical well- } \\
\text { being/safety, Threat to } \\
\text { emotional well-being/mental } \\
\text { health, Political repression/ } \\
\text { persecution }\end{array}$ \\
\hline Orr (2019) & Political Science & Various & Not explicitly specified & Focus group & $\begin{array}{l}\text { Sexual harassment/gender } \\
\text { discrimination, Political } \\
\text { repression/persecution, } \\
\text { Threat to physical well- } \\
\text { being/safety, Threat to } \\
\text { emotional well-being/mental } \\
\text { health }\end{array}$ \\
\hline Parkes (2010) & $\begin{array}{l}\text { Other Social Sciences/ } \\
\text { Interdisciplinary }\end{array}$ & South Africa & Not explicitly specified & Autoethnographic & Role conflicts \\
\hline Parkinson (2019) & Political Sciences & Iraq, Jordan & PI (non-local) & Autoethnographic & $\begin{array}{l}\text { Political repression/ } \\
\text { persecution, Role conflicts, } \\
\text { Poor employment/work } \\
\text { conditions }\end{array}$ \\
\hline Patel et al (2017) & Heath Sciences & India & Enumerators & Content analysis & $\begin{array}{l}\text { Role conflicts, Poor } \\
\text { employment/work } \\
\text { conditions }\end{array}$ \\
\hline
\end{tabular}


BMJ Global Health

Table 1 Continued

\begin{tabular}{|c|c|c|c|c|c|}
\hline Study reference & Scientific discipline & $\begin{array}{l}\text { Country of } \\
\text { study }\end{array}$ & $\begin{array}{l}\text { Subjects of ethical } \\
\text { assessment }\end{array}$ & Methodological approach & Ethical challenges \\
\hline $\begin{array}{l}\text { Pelcastre-Villafuerte et } \\
\text { al }(2015)^{64}\end{array}$ & Heath Sciences & Mexico & $\begin{array}{l}\text { PI (non-local), PhD } \\
\text { (non-local/local), Data } \\
\text { Collectors, RAs (gate } \\
\text { keepers...) (local) }\end{array}$ & Autoethnographic & $\begin{array}{l}\text { Threat to physical well- } \\
\text { being/safety, Poor } \\
\text { employment/work } \\
\text { conditions, Doubts about } \\
\text { purpose/legitimacy of } \\
\text { research/guilt }\end{array}$ \\
\hline $\begin{array}{l}\text { Peter and Strazzari } \\
(2017)\end{array}$ & Development Studies & Mali, Senegal & Not explicitly specified & Autoethnographic & $\begin{array}{l}\text { Threat to physical well- } \\
\text { being/safety, Limits of } \\
\text { IRBs/m } \\
\text { oral relativism }\end{array}$ \\
\hline
\end{tabular}

\begin{tabular}{lllll}
\hline Pio and Singh (2016) & Development Studies & Various & PI (non-local) & Autoethnographic \\
\hline Porter et al (2010) & Psychology & $\begin{array}{l}\text { Ghana, Malawi, } \\
\text { South Africa }\end{array}$ & $\begin{array}{l}\text { RAs (gate keepers...) } \\
\text { (local) }\end{array}$ & Autoethnographic
\end{tabular}

Threat to physical wellbeing/safety, Threat to emotional well-being/mental health South Africa (local)

Poor employment/work conditions, Unequal power dynamics, Threat to physical well-being/safety, Sexual harassment/gender discrimination

\begin{tabular}{|c|c|c|c|c|c|}
\hline Pyles (2015) & Social Work & Haiti & PI (non-local) & Autoethnographic & $\begin{array}{l}\text { Unequal power dynamics, } \\
\text { Sexual harassment/gender } \\
\text { discrimination, Role conflicts }\end{array}$ \\
\hline Raffety $(2015)^{31}$ & Anthropology/Ethnology & China & PI (non-local) & Autoethnographic & Role conflicts \\
\hline Råheim et al (2016) & Heath Sciences & Global & PI (non-local) & Autoethnographic & $\begin{array}{l}\text { Unequal power dynamics, } \\
\text { Threat to emotional well- } \\
\text { being/mental health, Doubts } \\
\text { about purpose/legitimacy of } \\
\text { research/guilt }\end{array}$ \\
\hline Rahmani et al (2015) & Heath Sciences & Iran & $\mathrm{PhD}$ (local) & Autoethnographic & Role conflicts \\
\hline Ralefala et al (2018) & $\begin{array}{l}\text { Other Social Sciences/ } \\
\text { Interdisciplinary }\end{array}$ & Botswana & PI (local) & Quantitative Survey & $\begin{array}{l}\text { Limits of IRBs/moral } \\
\text { relativism }\end{array}$ \\
\hline Reddy et al (2019) & Development Studies & South Sudan & $\begin{array}{l}\text { PI (non-local), RAs (gate } \\
\text { keepers...) (local) }\end{array}$ & $\begin{array}{l}\text { Autoethnographic, Qualitative } \\
\text { Interview }\end{array}$ & $\begin{array}{l}\text { Threat to emotional well- } \\
\text { being/mental health, Role } \\
\text { conflicts }\end{array}$ \\
\hline Reed (2002) & Political Science & $\begin{array}{l}\text { Liberia, Thailand, } \\
\text { Burma }\end{array}$ & Not explicitly specified & Autoethnographic & $\begin{array}{l}\text { Threat to emotional well- } \\
\text { being/mental health, Limits } \\
\text { of IRBs/moral relativism, } \\
\text { Threat to physical well- } \\
\text { being/safety }\end{array}$ \\
\hline Ridde et al (2019) & Heath Sciences & Global & PI (non-local) & Other & $\begin{array}{l}\text { Unequal power dynamics, } \\
\text { Sexual harassment/gender } \\
\text { discrimination }\end{array}$ \\
\hline Roberts et al (2019) & Heath Sciences & South Africa & Data Collectors & Qualitative Interview & $\begin{array}{l}\text { Threat to physical well- } \\
\text { being/safety, Threat to } \\
\text { emotional well-being/mental } \\
\text { health, Poor employment/ } \\
\text { work conditions, Role } \\
\text { conflicts }\end{array}$ \\
\hline Rogers-Brown (2015) ${ }^{78}$ & $\begin{array}{l}\text { Other Social Sciences/ } \\
\text { Interdisciplinary }\end{array}$ & Mexico & PI (non-local) & $\begin{array}{l}\text { Autoethnographic, Literature } \\
\text { Review }\end{array}$ & $\begin{array}{l}\text { Sexual harassment/gender } \\
\text { discrimination, Political } \\
\text { repression/persecution, Role } \\
\text { conflicts, Poor employment/ } \\
\text { work conditions, Threat to } \\
\text { emotional well-being/mental } \\
\text { health, Threat to physical } \\
\text { well-being/safety }\end{array}$ \\
\hline $\begin{array}{l}\text { Roll and Swenson } \\
(2019)^{79}\end{array}$ & Political Sciences & $\begin{array}{l}\text { Afghanistan, East } \\
\text { Timor }\end{array}$ & Not explicitly specified & Autoethnographic & $\begin{array}{l}\text { Threat to physical well- } \\
\text { being/safety, Role conflicts, } \\
\text { Sexual harassment/gender } \\
\text { discrimination, Political } \\
\text { repression/persecution, Role } \\
\text { conflicts }\end{array}$ \\
\hline Rossi (2004) & Development Studies & $\begin{array}{l}\text { West-African } \\
\text { Sahel State }\end{array}$ & Not explicitly specified & Autoethnographic & Unequal power dynamics \\
\hline
\end{tabular}

Continued 
Table 1 Continued

\begin{tabular}{|c|c|c|c|c|c|}
\hline Study reference & Scientific discipline & $\begin{array}{l}\text { Country of } \\
\text { study }\end{array}$ & $\begin{array}{l}\text { Subjects of ethical } \\
\text { assessment }\end{array}$ & Methodological approach & Ethical challenges \\
\hline $\begin{array}{l}\text { Rossouw and } \\
\text { Niemczyk (2013) }\end{array}$ & $\begin{array}{l}\text { Other Social Sciences/ } \\
\text { Interdisciplinary }\end{array}$ & South Africa & $\begin{array}{l}\text { RAs (gate keepers...) } \\
\text { (local) }\end{array}$ & Field Journal & $\begin{array}{l}\text { Poor employment/work } \\
\text { conditions, Unequal power } \\
\text { dynamics, Role conflicts }\end{array}$ \\
\hline Rothmann et al (2018) & Heath Sciences & Various & $\begin{array}{l}\text { PI (non-local), RAs (non- } \\
\text { local), Data Collectors }\end{array}$ & $\begin{array}{l}\text { Qualitative Interview, Focus } \\
\text { group }\end{array}$ & $\begin{array}{l}\text { Role conflicts, Threat to } \\
\text { emotional well-being/mental } \\
\text { health }\end{array}$ \\
\hline Roxburgh (2018) & $\begin{array}{l}\text { Other Social Sciences/ } \\
\text { Interdisciplinary }\end{array}$ & $\begin{array}{l}\text { Ghana, } \\
\text { Cameroon }\end{array}$ & $\begin{array}{l}\text { PhD (non-local), Data } \\
\text { Collectors }\end{array}$ & Autoethnographic & $\begin{array}{l}\text { Threat to emotional well- } \\
\text { being/mental health, Limits } \\
\text { of IRBs/moral relativism, } \\
\text { Sexual harassment/gender } \\
\text { discrimination }\end{array}$ \\
\hline $\begin{array}{l}\text { Salaam and Brown } \\
(2013)^{62}\end{array}$ & Psychology & Various & PI (non-local) & Autoethnographic & $\begin{array}{l}\text { Threat to physical well- } \\
\text { being/safety }\end{array}$ \\
\hline Schwedler $(2006)^{81}$ & Political Sciences & Jordan, Yemen & PI (non-local) & Autoethnographic & $\begin{array}{l}\text { Political repression/ } \\
\text { persecution, Unequal } \\
\text { power dynamics, Sexual } \\
\text { harassment/gender } \\
\text { discrimination }\end{array}$ \\
\hline Sharma $(2019)^{93}$ & $\begin{array}{l}\text { Geography/Agricultural } \\
\text { Science/Environmental } \\
\text { Science }\end{array}$ & India & PhD (non-local) & Field Journal & $\begin{array}{l}\text { Role conflicts, Unequal } \\
\text { power dynamics }\end{array}$ \\
\hline Shesterinina $(2019)^{77}$ & $\begin{array}{l}\text { Other Social Sciences/ } \\
\text { Interdisciplinary }\end{array}$ & Georgia & PI (non-local) & Qualitative Interview & $\begin{array}{l}\text { Threat to emotional well- } \\
\text { being/mental health, Role } \\
\text { conflicts, Threat to physical } \\
\text { well-being/safety, Political } \\
\text { repression/persecution }\end{array}$ \\
\hline $\begin{array}{l}\text { Sheyvens and Lesli } \\
\text { (2000) }\end{array}$ & Development Studies & Various & PI (non-local) & Literature Review & Unequal power dynamics \\
\hline $\begin{array}{l}\text { Shirmohammadi et al } \\
\text { (2018) }\end{array}$ & Psychology & Iran & PI (local), PhD (local) & Qualitative Interview & $\begin{array}{l}\text { Threat to emotional well- } \\
\text { being/mental health, } \\
\text { Poor employment/work } \\
\text { conditions, Limits of IRBs/ } \\
\text { moral relativism }\end{array}$ \\
\hline Shordike et al (2017) & $\begin{array}{l}\text { Other Social Sciences/ } \\
\text { Interdisciplinary }\end{array}$ & Thailand & PI (non-local) & Focus Group & $\begin{array}{l}\text { Poor employment/work } \\
\text { conditions, Unequal power } \\
\text { dynamics }\end{array}$ \\
\hline Sibai et al (2019) ${ }^{89}$ & Heath Sciences & Syria & Not explicitly specified & Autoethnographic & Unequal power dynamics \\
\hline $\begin{array}{l}\text { Silkin and Hendrie } \\
\text { (1997) }\end{array}$ & Political Science & Ethiopia, Eritrea & PI (non-local) & Autoethnographic & $\begin{array}{l}\text { Threat to physical well- } \\
\text { being/safety }\end{array}$ \\
\hline Simic (2016) & Sociology & $\begin{array}{l}\text { Bosnia } \\
\text { Herzegovina }\end{array}$ & PI (non-local) & Autoethnographic & $\begin{array}{l}\text { Threat to emotional well- } \\
\text { being/mental health, Role } \\
\text { conflicts, Doubts about } \\
\text { purpose/legitimacy of } \\
\text { research/guilt, Political } \\
\text { repression/persecution }\end{array}$ \\
\hline $\begin{array}{l}\text { Simon and Mosavel } \\
(2010)^{60}\end{array}$ & Heath Sciences & South Africa & $\begin{array}{l}\text { RAs (gate keepers...) } \\
\text { (local) }\end{array}$ & Autoethnographic & $\begin{array}{l}\text { Poor employment/work } \\
\text { conditions, Threat to } \\
\text { emotional well-being/mental } \\
\text { health }\end{array}$ \\
\hline Sinha (2017) & Heath Sciences & India & PhD (non-local) & Field Journal & $\begin{array}{l}\text { Threat to emotional well- } \\
\text { being/mental health, } \\
\text { Threat to physical } \\
\text { well-being/safety, } \\
\text { Doubts about purpose/ } \\
\text { legitimacy of research/ } \\
\text { guilt, Sexual harassment/ } \\
\text { gender discrimination, } \\
\text { Poor employment/work } \\
\text { conditions }\end{array}$ \\
\hline Smeltzer (2012) & Sociology & Malaysia & PI (non-local) & Autoethnographic & $\begin{array}{l}\text { Doubts about purpose/ } \\
\text { legitimacy of research/ } \\
\text { guilt, Political repression/ } \\
\text { persecution }\end{array}$ \\
\hline Smith et al (2014) ${ }^{94}$ & Heath Sciences & Global & PI (non-local), PI (local) & & Unequal power dynamics \\
\hline $\begin{array}{l}\text { Sowatey and Tankebe } \\
\text { (2018) }\end{array}$ & $\begin{array}{l}\text { Other Social Sciences/ } \\
\text { Interdisciplinary }\end{array}$ & Ghana & PI (non-local), PI (local) & Field Journal & $\begin{array}{l}\text { Threat to physical well- } \\
\text { being/safety }\end{array}$ \\
\hline
\end{tabular}


BMJ Global Health

Table 1 Continued

\begin{tabular}{lllll}
\hline Study reference & Scientific discipline & $\begin{array}{l}\text { Country of } \\
\text { study }\end{array}$ & $\begin{array}{l}\text { Subjects of ethical } \\
\text { assessment }\end{array}$ & Methodological approach \\
\hline Stodulka $(2015)^{29}$ & Heath Sciences & Indonesia & PI (non-local) & Autoethnographic \\
\hline Suen (2015) & $\begin{array}{l}\text { Other Social Sciences/ } \\
\text { Interdisciplinary }\end{array}$ & China & PI (non-local) & Autoethnographic
\end{tabular}

\begin{tabular}{lllll}
$\begin{array}{l}\text { Sukarieh and Tannock } \\
(2019)\end{array}$ & Anthropoligy/Ethnology & Syria & RAs (gate keepers...) & Qualitative Interview \\
\hline Sultana (2007) $)^{83}$ & $\begin{array}{l}\text { Other Social Sciences/ } \\
\text { Interdisciplinary }\end{array}$ & Bangladesh & Not explicitly specified & $\begin{array}{l}\text { Autoethnographic, Qualitative } \\
\text { Interview }\end{array}$
\end{tabular}

\begin{tabular}{|c|c|c|c|c|c|}
\hline $\begin{array}{l}\text { Sumner and Tribe } \\
(2008)^{97}\end{array}$ & Development Studies & Global & Not explicitly specified & Theoretical & $\begin{array}{l}\text { Unequal power dynamics. } \\
\text { Limits of IRBs/moral } \\
\text { relativism, Role conflicts. } \\
\text { Threat to physical well- } \\
\text { being/safety }\end{array}$ \\
\hline Theron (2016) & $\begin{array}{l}\text { Other Social Sciences/ } \\
\text { Interdisciplinary }\end{array}$ & South Africa & PI (local) & Autoethnographic & Role conflicts \\
\hline Thomson et al (2013) & $\begin{array}{l}\text { Other Social Sciences/ } \\
\text { Interdisciplinary }\end{array}$ & $\begin{array}{l}\text { Burundi, } \\
\text { Rwanda, DRC, } \\
\text { Uganda }\end{array}$ & Not explicitly specified & Autoethnographic & $\begin{array}{l}\text { Political repression/ } \\
\text { persecution, Role conflicts. } \\
\text { Threat to emotional well- } \\
\text { being/mental health, Limits } \\
\text { of IRBs/moral relativism, } \\
\text { Doubts about purpose/ } \\
\text { legitimacy of research/guilt }\end{array}$ \\
\hline Tsai $(2018)^{28}$ & Social Work & Philippines & Data Collectors & Field Journal & $\begin{array}{l}\text { Role conflicts, Threat to } \\
\text { emotional well-being/mental } \\
\text { health }\end{array}$ \\
\hline $\begin{array}{l}\text { Turcotte-Tremblay and } \\
\text { Mc Sween-Cadieux } \\
\text { (2018) }\end{array}$ & Heath Sciences & Various & PhD (non-local) & Autoethnographic & $\begin{array}{l}\text { Political repression/ } \\
\text { persecution }\end{array}$ \\
\hline Turner (2010) & $\begin{array}{l}\text { Other Social Sciences/ } \\
\text { Interdisciplinary }\end{array}$ & China, Vietnam & $\begin{array}{l}\text { PI (non-local), RAs (gate } \\
\text { keepers...) (local) }\end{array}$ & Qualitative Interview & $\begin{array}{l}\text { Unequal power dynamics, } \\
\text { Role conflicts, Poor } \\
\text { employment/work } \\
\text { conditions, Threat to } \\
\text { emotional well-being/mental } \\
\text { health }\end{array}$ \\
\hline Turner $(2013)^{22}$ & $\begin{array}{l}\text { Geography/Agricultural } \\
\text { Science/Environmental } \\
\text { Science }\end{array}$ & China, Vietnam & PI (non-local) & Autoethnographic & $\begin{array}{l}\text { Role conflicts, Political } \\
\text { repression/persecution, } \\
\text { Poor employment/work } \\
\text { conditions, Unequal } \\
\text { power dynamics, Sexual } \\
\text { harassment/gender } \\
\text { discrimination, Doubts } \\
\text { about purpose/legitimacy of } \\
\text { research/guilt }\end{array}$ \\
\hline Tyagi et al (2018) & $\begin{array}{l}\text { Other Social Sciences/ } \\
\text { Interdisciplinary }\end{array}$ & India & PI (local) & Field Journal & $\begin{array}{l}\text { Threat to emotional well- } \\
\text { being/mental health, Role } \\
\text { conflicts }\end{array}$ \\
\hline $\begin{array}{l}\text { van den Boogaard } \\
\text { (2019) }\end{array}$ & Development Studies & $\begin{array}{l}\text { Ghana, Sierra } \\
\text { Leone }\end{array}$ & PI (non-local) & Autoethnographic & $\begin{array}{l}\text { Unequal power dynamics, } \\
\text { Sexual harassment/gender } \\
\text { discrimination }\end{array}$ \\
\hline van Dijk $(2015)^{50}$ & $\begin{array}{l}\text { Other Social Sciences/ } \\
\text { Interdisciplinary }\end{array}$ & South Africa & PI (non-local) & Autoethnographic & $\begin{array}{l}\text { Doubts about purpose/ } \\
\text { legitimacy of research/guilt, } \\
\text { Role conflicts, Threat to } \\
\text { physical well-being/safety, } \\
\text { Unequal power dynamics, } \\
\text { Threat to emotional well- } \\
\text { being/mental health }\end{array}$ \\
\hline Vogler (2007) & $\begin{array}{l}\text { Other Social Sciences/ } \\
\text { Interdisciplinary }\end{array}$ & Thailand & PI (non-local) & Autoethnographic & $\begin{array}{l}\text { Political repression/ } \\
\text { persecution }\end{array}$ \\
\hline
\end{tabular}

Continued 
BMJ Global Health

\begin{tabular}{|c|c|c|c|c|c|}
\hline Study reference & Scientific discipline & $\begin{array}{l}\text { Country of } \\
\text { study }\end{array}$ & $\begin{array}{l}\text { Subjects of ethical } \\
\text { assessment }\end{array}$ & Methodological approach & Ethical challenges \\
\hline Vuong et al (2017) & Heath Sciences & Vietnam & PhD (local) & Quantitative Survey & $\begin{array}{l}\text { Political repression/ } \\
\text { persecution }\end{array}$ \\
\hline Wackenhut (2017) & Sociology & Egypt & $\mathrm{PhD}$ (non-local) & Autoethnographic & $\begin{array}{l}\text { Threat to physical well- } \\
\text { being/safety }\end{array}$ \\
\hline Wagman et al (2016) & Psychology & Uganda & PI (non-local) & Autoethnographic & Role conflicts \\
\hline Walsh et al (2016) $)^{91}$ & Heath Sciences & Zambia & $\begin{array}{l}\text { PI (non-local), PI (local), } \\
\text { RAs (gate keepers...) } \\
\text { (local), RAs (non-local) }\end{array}$ & Qualitative Interview & Unequal power dynamics \\
\hline Wamai (2014) & Political Sciences & Kenya & $\begin{array}{l}\text { RAs (gate keepers...) } \\
\text { (local), PhD (local) }\end{array}$ & Autoethnographic & $\begin{array}{l}\text { Sexual harassment/gender } \\
\text { discrimination, Threat to } \\
\text { physical well-being/safety, } \\
\text { Threat to emotional well- } \\
\text { being/mental health }\end{array}$ \\
\hline Williams $(2016)^{99}$ & Development Studies & Global & PI (non-local) & $\begin{array}{l}\text { Quantitative Survey, Qualitative } \\
\text { Interview, Autoethnographic, } \\
\text { Document Review, Literature } \\
\text { Review }\end{array}$ & $\begin{array}{l}\text { Limits of IRBs/moral } \\
\text { relativism }\end{array}$ \\
\hline Wilson et al (2018) & Health Sciences & Ghana & Not explicitly specified & Autoethnographic & $\begin{array}{l}\text { Poor employment/work } \\
\text { conditions, Role conflicts }\end{array}$ \\
\hline Wood $(2006)^{52}$ & Sociology & EI Salvador & PI (non-local) & Autoethnographic & $\begin{array}{l}\text { Threat to physical well- } \\
\text { being/safety, Role conflicts, } \\
\text { Threat to emotional well- } \\
\text { being/mental health, Doubts } \\
\text { about purpose/legitimacy of } \\
\text { research/guilt }\end{array}$ \\
\hline Wood $(2011)^{55}$ & Political Sciences & Global & PI (non-local) & Autoethnographic & $\begin{array}{l}\text { Threat to emotional well- } \\
\text { being/mental health, Threat } \\
\text { to physical well-being/ } \\
\text { safety, Role conflicts, } \\
\text { Doubts about purpose/ } \\
\text { legitimacy of research/guilt }\end{array}$ \\
\hline Woon $(2013)^{69}$ & Political Sciences & Philippines & PI (non-local) & Autoethnographic & $\begin{array}{l}\text { Threat to emotional well- } \\
\text { being/mental health, Threat } \\
\text { to physical well-being/ } \\
\text { safety, Role conflicts, } \\
\text { Doubts about purpose/ } \\
\text { legitimacy of research/guilt }\end{array}$ \\
\hline Yusupova (2019) & Political & Russia & PI (non-local) & Autoethnographic & $\begin{array}{l}\text { Role conflicts, Political } \\
\text { repression/persecution }\end{array}$ \\
\hline Zulu et al (2018) & Heath Sciences & Global & Not explicitly specified & Autoethnographic & Role conflicts \\
\hline Zwi et al (2006) & Heath Sciences & Global & PI (non-local) & Autoethnographic & $\begin{array}{l}\text { Doubts about purpose/ } \\
\text { legitimacy of research/guilt, } \\
\text { Threat to emotional well- } \\
\text { being/mental health, Role } \\
\text { conflicts }\end{array}$ \\
\hline
\end{tabular}

IRBs, institutional review boards; $\mathrm{PI}$, principal investigator; RA, research assistant.

the 'harrowing testimonies of individuals who had survived a massacre' and 'children nearby [...] dying from cholera'. ${ }^{42}$ Exposure to traumatic narratives, stories and images can have substantial emotional ramifications for research staff. Here, several included studies highlighted researchers' reports of vicarious and secondary trauma. ${ }^{283244475455}$ Further emotional reactions included anxiety and fear, ${ }^{56-58}$ insomnia, ${ }^{56} 59$ 60 ${ }^{6}$ epression, ${ }^{55} 60$ burn-out, ${ }^{55} 61$ as well as social isolation. ${ }^{4655}$ Local data collectors are often instructed to treat study participants with empathy and compassion in order to gain their trust and to encourage the disclosure of potential traumatic experiences. ${ }^{24} 38606263$ In consequence, it is difficult for them to maintain an adequate level of emotional distance as emphasised by a data collector in an included qualitative study: 'whenever they cry, I cry with them'. 63

\section{Physical safety risks}

Of 183, 48 (26\%) studies discussed how data collection in LMICs can expose research staff to substantial physical safety threats. Several included studies highlighted security risks associated with harsh weather conditions and natural disasters. ${ }^{186465}$ Included studies also pointed to risks associated with travelling to research sites, including traffic accidents ${ }^{26}$ becoming caught up in political protests, ${ }^{5766}$ or being stopped and interrogated by soldiers or rebels. ${ }^{62}{ }^{67}$ Data collection can be particularly dangerous when implemented in settings with high levels of crime,${ }^{3368}$ gang violence, ${ }^{3357}$ terrorism incidents, ${ }^{6970}$ or 


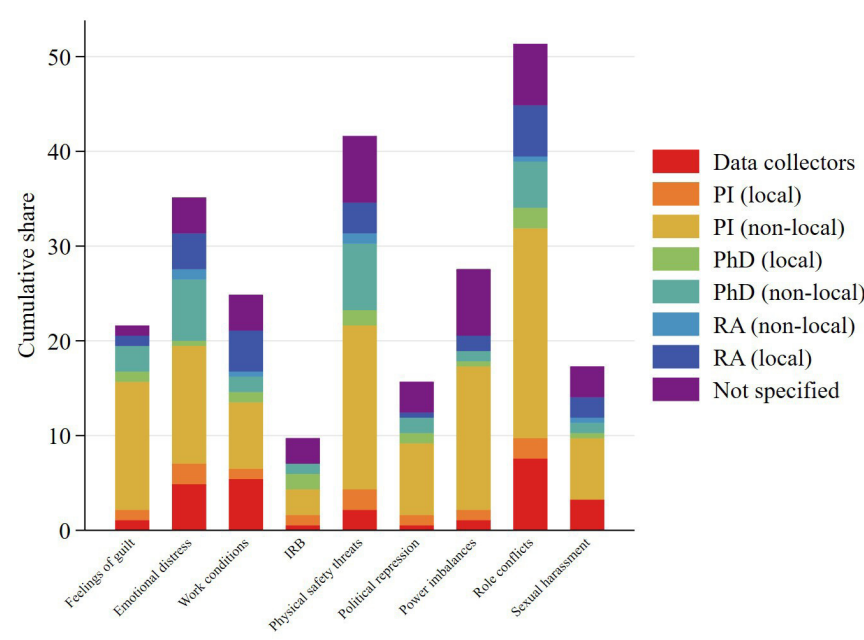

Figure 3 Ethical challenges and affected research staff. IRB, institutional review boards; PI, principal investigator; RA, research assistant.

in postconflict contexts exerting elevated levels of political instability. ${ }^{71}$ In three included studies from Iraq, Afghanistan, and Palestine, data collection had to be put on hold intermittently due to political violence and bombings. ${ }^{15} 4272$ Local data collectors are particularly vulnerable to security risks during their visits to the study site and to participants' homes. ${ }^{39}$ Non-local research staff, on the other hand, will likely be less familiar with specific security threats and necessary safety precautions and may additionally have a high visibility due to their foreigner status. ${ }^{72}$ Similarly, local researchers may also be asked to collect data in settings that they are not necessarily familiar and comfortable with, including for examples vulnerable and marginalised communities, ${ }^{50}$ hospitals ${ }^{73}$ or displaced person camps, ${ }^{42}$ as well as in locations that deviate from their own in terms of language, ethnicity, culture or religion. ${ }^{74-76}$ One study suggested that project timelines, interview targets and budget constraints may further lever out necessary security precautions. ${ }^{77} 78$

\section{Political Repression and Persecution}

Relatedly, 27/183 (15\%) studies identified political repression and persecution as ethical challenges of data collection activities, the scope of which may include imprisonment, ${ }^{77} 78$ attempts of intimidation and harassment by police officers or local authorities ${ }^{79}$ accusations and suspicion by local community members ${ }^{53} 80$ and seizure of collected data ${ }^{80}$ Non-local researchers further reported cases of travel bans to their research site and confiscation of their identification documents. ${ }^{80}$ However, while in many cases they are able to 'evacuate rapidly, local interlocutors rarely can'. ${ }^{15}$ Data collection in autocratic countries increases the risk of political persecution, particularly when insurgents or protesters are interviewed and politically sensitive information is disclosed in the process. ${ }^{78798182}$

\section{Sexual harassment}

Of 183, $20(11 \%)$ studies mentioned sexual harassment as another ethical challenge that particularly affects female research staff. Included studies listed accounts of 'sexual objectification', 33 'unpleasant requests', 8384 or 'sexually offensive' messages and encounters experienced during data collection. ${ }^{85}$ The threat from sexual harassment may further be exacerbated if the work is carried out during hours of darkness and when female data collectors conduct interviews alone in the homes of male participants. ${ }^{58} 80$ The vulnerability of female researchers may also be amplified in interactions with male gatekeepers who may be able to abuse given dependency and power dynamics to demonstrate their 'status and masculine dominance ${ }^{, 79}$ or to reject female researchers as 'a glaring threat to patriarchal norms'. ${ }^{86}$

\section{Inadequate working conditions}

Ambitious timelines, inflexible research budgets and prescribed recruitment and interview targets for data collection may increase the risk of inadequate working conditions, as outlined by 48/183 (26\%) studies. High workloads are particularly problematic as this may increase staffers' risk for burnout (see above).$^{23248788}$ In addition, studies noted that local research staff members are often recruited in contexts of widespread poverty and unemployment and may therefore be compelled to accept low remuneration and short-term contracts. ${ }^{3760} 8488$ Due to time constraints and the specific nature of incentive structures, lead researchers (PIs) are often not physically present at the study site and may thus find it difficult to effectively appraise local conditions and to provide local staff members with adequate support. Provisional project structures and intercultural communication gaps may further negatively affect working conditions. ${ }^{84} 87$ Moreover, studies pointed to hostilities and accusations made by local community members, ${ }^{25}$ and to the physical burden of data collection linked to travelling long distances by foot. ${ }^{54}$

\section{North-South power imbalances}

Closely linked to inadequate working conditions are discriminatory power imbalances in multinational research teams, which were cited in 52/183 (28\%) studies. Several included studies referenced the terms 'helicopter research' and 'extractive science' to describe dynamics in which researchers from high-income countries set out to collect data in LMICs without adequately giving credit to local contributions and knowledge and without engaging in meaningful capacity building efforts. ${ }^{15}{ }^{89-91}$ Non-local researchers may also fail to actively involve local research partners in knowledge production processes. ${ }^{90}$ In consequence, the research process can foster dominance of high-income countries in academia, may fuel inequities in authorships and exploit intellectual contributions of researchers from LMICs. ${ }^{36} 84$ 91-94 Some studies voiced concerns that research in LMICs may perpetuate the prevailing structural power imbalances of the NorthSouth dichotomy and culminate in racism and neocolonialism. ${ }^{95} 96$ 


\section{Limited responsiveness of institutional review boards}

Lastly, PIs from high-income countries and their academic institutions are often unfamiliar with the specific study setting in LMICs. Relatedly, 18/183 (10\%) studies discussed contextual ethical dilemmas and the potentially problematic role of institutional review boards (IRBs). While guidance from IRBs can be an asset, it may also become a burden if guidelines contradict each other or do not correspond to the reality of the local research setting. In this regard, the fundamental question remains as to whether 'universal or local ethics (should) be adopted ${ }^{97}$ and how Western ethics principles may fail to be 'applicable in (the) cultures' they target. ${ }^{98}$ These are likely more pronounced when IRBs do 'not have the necessary expertise in the international development context'.99

\section{DISCUSSION}

This is the first systematic review to examine the ethical challenges faced by research staff across various disciplines when conducting human subjects research in LMICs. The identified challenges were interlinked, multifaceted and affected both local and non-local research staff across all power hierarchies. Listed challenges comprised threats to physical safety, particularly in settings offering up high levels of political instability and emotional harm, in many cases associated with exposure to participants' experiences of trauma, violence, disaster or economic destitution. The emotional distress experienced by research staff members can further be exacerbated by factors such as working overtime, concerns about participants' wellbeing and role conflicts. As a result of unresolved role conflicts, research staff can internalise feelings of guilt and shame. While local RAs and data collectors play a central role in securing access to study participants and ensuring the cultural adequacy of study materials, they are particularly prone to being subjected to exploitative working conditions and inequities in scientific acknowledgement.

This systematic review emphasises the severity of ethical challenges affecting research staff 'in the field'. This stands in stark contrast to the scope and function of existing ethics guidelines and IRBs, which largely neglect the issue of protecting research staff altogether, particularly when it comes to the ethical standards and needs in host countries. Current ethics guidelines and regulations often do not take into account specific conditions found in local research settings, which makes these less suited for supporting and protecting research staff 'in the field'. ${ }^{11} 1987100$ Irrespective of that, there are several strategies that individual researchers could implement to alleviate the excessive strains put on research staff and thus increase the ethical integrity of research projects. Regular debriefings with trained counsellors or psychologists to prevent the risk of vicarious traumatisation and compassion fatigue are desirable, and informal counselling with (senior) colleagues can also help to process emotional and psychological strains. ${ }^{29} 445097$ Another protective measure could stem from close collaboration with the existing social service infrastructure in a given research environment in order to institutionalise referrals of participants who are at high risk of harm and thereby relieve part of the emotional burden and responsibility that may otherwise be shouldered by research staff. $^{28} 42100$ Importantly, such protection and support structures are not meant to alienate researchers from the 'field' and from the lived experiences of their research participants altogether. 'Empathetic witnessing' can be a valuable component of scientific knowledge generation as long as the psychological well-being of research staff is not harmed.Research endeavours in conflict-prone settings may benefit from comprehensive risk assessments and consultations with local experts prior to data collection. ${ }^{59} 101102$ Lastly, meaningful capacity building by ways of engaging local research partners as coauthors or by offering vocational training programmes may help rectify existing power imbalances between research staff from high-income countries and LMICs. ${ }^{94} 103$ More importantly, local research collaborators should be engaged as equal partners and be involved at all stages of the research project so as to ensure that the formulation of research questions and hypotheses as well as the analysis and interpretation of findings is built on profound contextual and cultural knowledge. ${ }^{1572} 76$ Overall, formalised protection mechanisms are urgently needed and could be most effectively developed in dialogue with stakeholders across hierarchies and involving researchers irrespective of their country of origin equally. Given dilemmas of collective action and power asymmetries, structural barriers must be overcome particularly urgently, which could be realised, for example, by providing specific funding to the relevant bodies, universities, IRBs and research ministries. In the long term, policy-makers may create more tangible improvements by translating the debate into institutional change in terms of international agreements and national laws. Lastly, the scientific community should engage in a critical reflection on the term of 'field' research as such and problematise its inherent notion of 'othering' and 'exoticising' research settings—as well as 'local' research staff-in the Global South.

A major strength of this study is its broad scope and the large number of studies that were assessed and synthesised. Based on this wide evidence base, we are able to generate findings that carry relevance and implications across social science disciplines. Another strength of this review is the elaboration of a categorisation scheme for the ethical challenges faced by research staff, developed in an iterative, consensual process between the study authors. This scheme can serve as an essential basis for integrating safeguarding mechanisms in future research projects and for adapting ethics guidelines and IRB assessments to the different kinds of ethical challenges presented here. Yet, while IRB guidelines should be guided by the overarching principles of respect, justice and beneficence, they should also aim to be situated and 
practical, and thus tailored and responsive to each particular case and setting. ${ }^{104}$

Some gaps and limitations of this review are noteworthy. First, the majority of included studies discussed challenges faced by research staff members on higher hierarchical levels, namely by PIs or PhD students. While we have outlined possible heterogeneities in the risks and challenges faced by research staff across different hierarchical levels, these observations may suffer from an under-representation of the perspectives and voices of research staff at lower levels and thus a somewhat biased perspective. Engaging local research staff-including data collectors, RAs, and gatekeepers-in an open dialogue is necessary to better represent their perspectives and identify potential additional ethical frictions. Second our systematic review does not exhaustively cover all research staff groups. For example, it excludes experiences that might be unique to postdoctoral researchers on short-term contracts and under high publication pressure, where it is reasonable to assume that both aspects could potentially incentivise engagement in high-risk research projects. Third, our systematic review only included studies that were published in English and may thus be biased towards a more Western, high-income perspective. Third, the search strategy was set up to prioritise scientific outlets and we may therefore have systematically neglected more informal outlets such as blogposts, radio broadcasts, tweets and local news outlets. These, however, may more adequately capture the perspectives of academically disempowered research staff from LMICs, who are particularly likely to make use of these channels. Future research should aim to shed light on researchers' experiences in a more comprehensive, and potentially more representative, manner. For instance, qualitative interviews with local research staff or largescale surveys targeting all hierarchical layers of a research team could help to shed light on these under-represented perspectives. Finally, most included studies relied on autoethnographic methodology, thus generating quite context-specific, singular data and insights. While methodological peculiarities may induce a stronger interaction of these disciplines with 'the field', the selectivity of reporting may also point to a gap in awareness across disciplines. Assuming that several of the ethical challenges sketched in this review apply to data collection in LMICs more broadly (though to different extents), more training and interdisciplinary exchange on reflexivity and positionality may be required. As suggested previously, more comprehensive reporting rules on ethical aspects could additionally help to increase such reflection.

Research staff play a pivotal role in guaranteeing the success of any research endeavour. The UN Sustainable Development Goal 8.8 aims to "protect labour rights and promote safe and secure working environments for all'. In order to prevent social science research from violating this very goal, the guiding ethical principle of 'do no harm' should equally apply to any research staff involved. The current COVID-19 pandemic is projected to increase global inequalities and put further strains on working conditions and employment security worldwide. Addressing the complex ethical challenges identified in this review has thus become more important than ever. While we have provided a first set of clear-cut evidence, more research is needed to guide global action and international research collaboration.

\section{Author affiliations}

${ }^{1}$ TUM School of Governance, Technical University of Munich, Munich, Germany ${ }^{2}$ Department of Social Policy and Intervention, University of Oxford, Oxford, UK ${ }^{3}$ Independent Development Evaluation (IDEV), African Development Bank (AfDB), Abidjan, COTE D'IVOIRE

${ }^{4}$ University of Cologne, Koeln, Germany

${ }^{5}$ German Development Institute, Bonn, Germany

${ }^{6}$ University of Göttingen, Gottingen, Germany

${ }^{7}$ Institute for the World Economy, Kiel, Germany

Twitter Janina Isabel Steinert @jisteinert, David Atika Nyarige @david_atika, Milan Jacobi @Milan_Jacobi, Jana Kuhnt @JanaKuhnt and Lennart Kaplan @LennartKap

Acknowledgements We are grateful to Gergana Manalova for her contributions to this systematic review, to Ana Garcia Hernandez for her conceptual input to the wider research project, and to Hannah Prince for proofreading. We are also thankful for financial and logistic support provided by the German Development Institute and the Joachim Herz Foundation. Moreover, constructive comments from participants at the DIE brownbag seminar series, the Global Dev9, and the DEval and Campbell Collaboration's Conference 2019, the European Political S elopment Conference 201cience Association's Annual Conference 2020 and the C4ED brownbag seminar series helped to improve this review considerably.

Contributors JS, JK and LK conceptualised and led the study. JS, JK and LK developed the review protocol, ran searches, screened abstracts and titles, and extracted and analysed the data with feedback from all authors. MJ and DAN screened articles, extracted the data and contributed to the interpretation of findings. JS drafted the first version of the manuscript and JK and LK provided substantial revisions and feedback. All authors have read and approved the final version of the manuscript.

Funding German Development Institute/Deutsches Institut fuer Entwicklungspolitik (DIE) \& Joachim-Herz Foundation.

Map disclaimer The inclusion of any map (including the depiction of any boundaries therein), or of any geographic or locational reference, does not imply the expression of any opinion whatsoever on the part of BMJ concerning the legal status of any country, territory, jurisdiction or area or of its authorities. Any such expression remains solely that of the relevant source and is not endorsed by BMJ. Maps are provided without any warranty of any kind, either express or implied.

Competing interests None declared.

Patient consent for publication Not required.

Provenance and peer review Not commissioned; externally peer reviewed.

Data availability statement Data are available on request. Data extraction sheets as well as detailed search strings for each database will be shared via https://osf. io/cjfbh/.

Supplemental material This content has been supplied by the author(s). It has not been vetted by BMJ Publishing Group Limited (BMJ) and may not have been peer-reviewed. Any opinions or recommendations discussed are solely those of the author(s) and are not endorsed by BMJ. BMJ disclaims all liability and responsibility arising from any reliance placed on the content. Where the content includes any translated material, BMJ does not warrant the accuracy and reliability of the translations (including but not limited to local regulations, clinical guidelines, terminology, drug names and drug dosages), and is not responsible for any error and/or omissions arising from translation and adaptation or otherwise.

Open access This is an open access article distributed in accordance with the Creative Commons Attribution Non Commercial (CC BY-NC 4.0) license, which permits others to distribute, remix, adapt, build upon this work non-commercially, and license their derivative works on different terms, provided the original work is properly cited, appropriate credit is given, any changes made indicated, and the use is non-commercial. See: http://creativecommons.org/licenses/by-nc/4.0/. 
ORCID iD

Janina Isabel Steinert http://orcid.org/0000-0001-7120-0075

\section{REFERENCES}

1 Hazelgrove J. The old faith and the new science: the Nuremberg code and human experimentation ethics in Britain, 1946-73. Soc Hist Med 2002;15:109-35.

2 Israel M, Hay I. Research ethics for social scientists. SAGE, 2006.

3 Brandt AM. Racism and research: the case of the tuskegee syphilis study. Hastings Cent Rep 1978;8:21-9.

4 Zimbardo PG. On the ethics of intervention in human psychologica research: with special reference to the Stanford prison experiment. Cognition 1973;2:243-56.

5 Holden C. Ethics in social science research. Science 1979;206:537-8

6 Lenza M. Controversies surrounding Laud Humphreys' tearoom trade: an unsettling example of politics and power in methodological critiques. Int J Sociol Soc Policy 2004;24:20-31.

7 Sales BD, Folkman S. Ethics in research with human participants. American Psychological Association, 2000.

8 Flicker S, Travers R, Guta A, et al. Ethical dilemmas in communitybased participatory research: recommendations for institutional review boards. J Urban Health 2007;84:478-93.

9 Chiseri-Strater E. Turning in upon ourselves: Positionality, subjectivity, in case study and ethnographic research. Ethics and Representation in Qualitative Studies of Literacy:115-33.

10 Schroeder D, Chatfield K, Singh M. Equitable research partnerships: a global code of conduct to counter ethics dumping. Springer International Publishing, 2019

11 Kaplan L, Kuhnt J, Steinert JI. Do no harm? field research in the global South: ethical challenges faced by research staff. World Dev 2020;127:104810.

12 Casale MAJ, Flicker S, Nixon SA. Fieldwork challenges: lessons learned from a north-south public health research partnership. Health Promot Pract 2011;12:734-43.

13 White $\mathrm{H}$. The twenty-first century experimenting Society: the four waves of the evidence revolution. Palgrave Commun 2019;5:1-7.

14 Humphreys M. Reflections on the ethics of social experimentation. J Glob Dev 2015:6:87-112.

15 Cronin-Furman K, Lake M. Ethics abroad: fieldwork in fragile and violent contexts. PS 2018;51:607-14.

16 Scheyvens R, Leslie H. Gender, ethics and empowerment: dilemmas of development fieldwork. Women's Studies International Forum 2000;23:119-30.

17 Belur J. Status, gender and geography: power negotiations in police research. Qualitative Research 2014;14:184-200.

18 Bachmann V. Participating and observing: positionality and fieldwork relations during Kenya's post-election crisis. Area 2011;43:362-8.

19 Hamid MO. Fieldwork for language education research in rural Bangladesh: ethical issues and dilemmas. Int $J$ Res Method Educ 2010;33:259-71.

20 Rahmani A, Merghati-Khoei E, Moghaddam-Banaem L, et al. Sexuality research in Iran: a focus on methodological and ethical considerations. Iran J Public Health 2015;44:979-86.

21 Nama N, Swartz L. Ethical and social dilemmas in communitybased controlled trials in situations of poverty: a view from a South African project. J Community Appl Soc Psychol 2002;12:286-97.

22 Turner S. Red stamps and green tea: fieldwork negotiations and dilemmas in the Sino-Vietnamese borderlands, 2013.

23 Kingori P, Gerrets R. Morals, morale and motivations in data fabrication: medical research fieldworkers views and practices in two sub-Saharan African contexts. Soc Sci Med 2016;166:150-9.

24 Kamuya DM, Marsh V, Njuguna P, et al. "When they see us, it's like they have seen the benefits!": experiences of study benefits negotiations in community-based studies on the Kenyan Coast. BMC Med Ethics 2014;15:90.

25 Madiega PA, Jones G, Prince RJ, et al. 'She's my sister-in-law, my visitor, my Friend' -- challenges of staff identity in home follow-up in an HIV trial in Western Kenya. Dev World Bioeth 2013;13:21-9.

26 Canavati SE, Quintero CE, Haller B, et al. Maximizing research study effectiveness in malaria elimination settings: a mixed methods study to capture the experiences of field-based staff. Malar J 2017:16:362

27 Einarsdóttir J. Child survival in affluence and poverty: ethics and fieldwork experiences from Iceland and Guinea-Bissau. Field methods 2006;18:189-204.
28 Cordisco Tsai L, Tsai LC. Conducting research with survivors of sex trafficking: lessons from a financial diaries study in the Philippines. Br J Soc Work 2018;48:158-75.

29 Stodulka T. Emotion work, ethnography, and survival strategies on the streets of Yogyakarta. Med Anthropol 2015;34:84-97.

30 D'souza NA, Guzder J, Hickling F, et al. The ethics of relationality in implementation and evaluation research in global health: reflections from the Dream-A-World program in Kingston, Jamaica. BMC Med Ethics 2018;19:50.

31 Raffety EL. Minimizing social distance: participatory research with children. Childhood 2015;22:409-22.

32 Rothman EF, Farrell A, Bright K, et al. Ethical and practical considerations for collecting research-related data from commercially sexually exploited children. Behav Med 2018;44:250-8.

33 Pawelz J. Researching gangs: how to reach hard-to-reach populations and negotiate tricky issues in the field. Forum Qualitative Sozialforschung 2018;19.

34 Gerharz E. Navigating unpredictable sites: methodological implications of positioning during and after fieldwork in conflict societies. Soc Anal 2017;61:1-18.

35 Dickson-Swift V, James EL, Kippen S. Do university ethics committees adequately protect public health researchers? Aust N Z J Public Health 2005;29:576-9.

36 Shordike A, Hocking C, Bunrayong W, et al. Research as relationship: engaging with ethical intent. Int J Soc Res Methodol 2017;20:285-98.

37 Mosavel M, Simon C, van Stade D, et al. Community-Based participatory research (CBPR) in South Africa: engaging multiple constituents to shape the research question. Soc Sci Med 2005;61:2577-87.

38 Devries KM, Child JC, Elbourne D, et al. "I never expected that it would happen, coming to ask me such questions":Ethical aspects of asking children about violence in resource poor settings. Trials 2015;16:516.

39 Roberts K, Gordon S, Sherr L, et al. 'When you are a data collector you must expect anything'. barriers, boundaries and breakthroughs: insights from the South African data-collection experience. Glob Health Promot 2020;27:54-62.

40 Gheondea-Eladi A. Health research ethics: between ethics codes and culture. J Empir Res Hum Res Ethics 2017;12:246-60.

41 Wagman JA, Paul A, Namatovu F, et al. Ethical challenges of randomized violence intervention trials: examining the share intervention in Rakai, Uganda. Psychol Violence 2016;6:442-51.

42 Browne B, Moffett L. Finding your feet in the field: critical reflections of early career researchers on field research in transitional societies. J Hum Rights Pract 2014;6:223-37.

43 Berghs M. Coming to terms with inequality and exploitation in an African state: researching disability in Sierra Leone. Disabil Soc 2010;25:861-5.

44 Coles J, Astbury J, Dartnall E, et al. A qualitative exploration of researcher trauma and researchers' responses to investigating sexual violence. Violence Against Women 2014;20:95-117.

45 Augustin A-LA. Rumours, fears and solidarity in fieldwork in times of political turmoil on the verge of war in southern Yemen. Contemp Soc Sci 2018;13:444-56.

46 Calgaro E. If you are vulnerable and you know it raise your hand: experiences from working in post-tsunami Thailand. Emot Space Soc 2015;17:45-54.

47 Dominey-Howes D. Seeing 'the dark passenger' - reflections on the emotional trauma of conducting post-disaster research. Emot Space Soc 2015;17:55-62.

48 Maier SL, Monahan BA. How close is too close? balancing Closeness and detachment in qualitative research. Deviant Behav 2009;31:1-32.

49 Mayan MJ, Daum CH. Worth the risk? Muddled relationships in community-based participatory research. Qual Health Res 2016;26:69-76

50 Dijk Dvan. Mission Impossible: Not Getting Emotionally Involved in Research among Vulnerable Youth in South Africa 2 This chapter stems from work on a PhD thesis. In: Contributions from European symbolic Interactionists: reflections on methods. Emerald Group Publishing Limited, 2015: 61-77. https://openaccess.leidenuniv.nl/ handle/1887/13382

51 Groes-Green C. Ambivalent participation: sex, power, and the anthropologist in Mozambique. Med Anthropol 2012;31:44-60.

52 Wood EJ. The ethical challenges of field research in conflict zones. Qual Sociol 2006;29:373-86.

53 Kunnath GJ. Anthropology's Ethical Dilemmas: Reflections from the Maoist Fields of India. Current Anthropology 2013;54:740-52. 
54 Mosavel M, Ahmed R, Daniels D, et al. Community researchers conducting health disparities research: ethical and other insights from fieldwork journaling. Soc Sci Med 2011;73:145-52.

55 Wood EJ. Reflections on the challenges, dilemmas, and rewards of research in conflict zones. In: Research methods in conflict settings: a view from below, 2011: 295-308.

56 Klocker N. Participatory action research: the distress of (not) making a difference. Emot Space Soc 2015;17:37-44.

57 Gokah T. The naïve researcher: doing social research in Africa. Int $J$ Soc Res Methodol 2006;9:61-73.

58 Derry P, Baum A. The role of the experimenter in field studies of distressed populations. J Trauma Stress 1994;7:625-35.

59 Moss SM, Uluğ Özden Melis, Acar YG. Doing research in conflict contexts: practical and ethical challenges for researchers when conducting fieldwork. Peace Confl 2019;25:86-99.

60 Simon C, Mosavel M. Community members as recruiters of human subjects: ethical considerations. Am J Bioeth 2010;10:3-11.

61 True G, Alexander LB, Fisher CB. Supporting the role of community members employed as research staff: perspectives of community researchers working in addiction research. Soc Sci Med 2017:187:67-75.

62 Salaam AO, Brown J. Ethical dilemmas in psychological research with vulnerable groups in Africa. Ethics Behav 2013;23:167-78.

63 Gouda HN, Kelly-Hanku A, Wilson L, et al. "Whenever they cry, I cry with them": Reciprocal relationships and the role of ethics in a verbal autopsy study in Papua New Guinea. Soc Sci Med 2016;163:1-9.

64 Pelcastre-Villafuerte BE, Domínguez-Esponda R, Vázquez TTG. Health social research in a context of violence: a look from the ethics. Saude e Sociedade 2015;24:816-25.

$65 \mathrm{Hu}$ M. Challenges of conducting disaster research: the case of the Sichuan earthquake. Risk Hazards Crisis Public Policy 2015;6:164-82.

66 Ghosh D. Risky fieldwork: the problems of ethics in the field. Energy Res Soc Sci 2018;45:348-54.

67 Castillo RC. The emotional, political, and analytical labor of engaged anthropology amidst violent political conflict. Med Anthropol 2015;34:70-83.

68 Belousov K, Horlick-Jones T, Bloor M, et al. Any Port in a storm: fieldwork difficulties in dangerous and crisis-ridden settings. Qualitative Research 2007;7:155-75.

69 Woon CY. For 'emotional fieldwork' in critical geopolitical research on violence and terrorism. Polit Geogr 2013;33:31-41.

70 Chaitin J. "I wish he hadn't told me that": methodological and ethical issues in social trauma and conflict research. Qual Health Res 2003;13:1145-54.

71 Neto PF. Surreptitious ethnography: following the paths of Angolan refugees and returnees in the Angola-Zambia borderlands. Ethnography 2019;20:128-45.

72 Dodsworth S. The challenges of making research collaboration in Africa more equitable. Oxford research encyclopedia of politics, 2019. https://oxfordre.com/politics/view/10.1093/acrefore/ 9780190228637.001.0001/acrefore-9780190228637-e-1389

73 Jepkosgei J, Nzinga J, McKnight J. Maintaining distance and staying immersed: practical ethics in an Underresourced new born unit. J Empir Res Hum Res Ethics 2019;14:509-12.

74 Kamuya DM, Molyneux CS, Theobald S. Gendered negotiations for research participation in community-based studies: implications for health research policy and practice. BMJ Glob Health 2017;2:e000320.

75 Molony T, Hammett D. The friendly Financier: talking money with the silenced assistant. Hum Organ 2007;66:292-300.

76 Molyneux S, Kamuya D, Marsh V. Community members employed on research projects face crucial, often under-recognized, ethical dilemmas. Am J Bioeth 2010;10:24-6.

77 Shesterinina A. Ethics, empathy, and fear in research on violent conflict. J Peace Res 2019;56:190-202.

78 Rogers-Brown JB. More than a war story: a feminist analysis of doing dangerous fieldwork. Advances in Gender Research 2015;20:111-31.

79 Roll K, Swenson G. Fieldwork after conflict: contextualising the challenges of access and data quality. Disasters 2019;43:240-60.
80 Mwambari D. Local Positionality in the production of knowledge in northern Uganda. Int J Qual Methods 2019;18:160940691986484.

81 Schwedler J. The third gender: Western female researchers in the middle East. PS: Political Science and Politics 2006:39:425-8.

82 Thomson S, Ansoms A, Murison J. Emotional and ethical challenges for field research in Africa: the story behind the findings. Palgrave Macmillan UK, 2013.

83 Sultana F. Reflexivity, positionality and participatory ethics: negotiating fieldwork dilemmas in international research. ACME 2007:6:374-85.

84 Rossouw JP, Niemczyk EK. A dual perspective on risks and security within research assistantships. J Res Pract 2013;9:7.

85 Grünenfelder J. A foreign woman researcher in a Purdah Society: opportunities and challenges for knowledge production in the 2000s. Hum Organ 2014;73:214-23.

86 Sultana S, Guimbretière F, Sengers P. Design within a patriarchal Society: opportunities and challenges in designing for rural women in Bangladesh, 2018.

87 Jayawickrama J. 'If They can't do any Good, They shouldn't Come': Northern Evaluators in Southern Realities. J Peacebuilding Dev 2013;8:26-41.

88 Molyneux S, Kamuya D, Madiega PA, et al. Field workers at the interface. Dev World Bioeth 2013;13:ii-iv.

89 Sibai AM, Rizk A, Coutts AP, et al. North-South inequities in research collaboration in humanitarian and conflict contexts. Lancet 2019;394:1597-600.

90 Lansford JE, Gauvain M, Koller SH, et al. The importance of international collaborative research for advancing understanding of child and youth development. International Perspectives in Psychology 2019;8:1-13.

91 Walsh A, Brugha R, Byrne E. "The way the country has been carved up by researchers": ethics and power in north-south public health research. Int J Equity Health 2016;15:204.

92 Abimbola S. The foreign gaze: authorship in academic global health. BMJ Glob Health 2019;4:e002068.

93 Sharma S. Me again: fieldwork, practice and returning. Area 2019;51:508-15.

94 Smith E, Hunt M, Master Z. Authorship ethics in global health research partnerships between researchers from low or middle income countries and high income countries. BMC Med Ethics 2014;15:42.

95 Mwambari D. Africa's next decolonisation battle should be about knowledge. Available: https://www.aljazeera.com/ indepth/opinion/africa-decolonisation-battle-knowledge190906074211760.html

96 Contemporary research must stop relying on racial inequalities. Africa at LSE, 2020. Available: https://blogs.Ise.ac.uk/africaatlse/ 2020/01/30/research-must-stop-racial-inequalities-colonialism/

97 Sumner A, Tribe M. International development studies: theories and methods in research and practice. SAGE Publications Ltd, 2008.

98 Makhoul J, El-Alti L, Qutteina Y, et al. "Protecting" or "Policing": Academic Researchers' View of IRBs in an Arab Context. J Empir Res Hum Res Ethics 2014:9:25-35.

99 Groves Williams L, Williams LG. Ethics in international development evaluation and research: what is the problem, why does it matter and what can we do about it? J Dev Effect 2016;8:535-52.

100 Sibai AM, Rizk A, Coutts AP, et al. North-South inequities in research collaboration in humanitarian and conflict contexts. The Lancet 2019;394:1597-600.

101 Kovats-Bernat JC. Factional terror, Paramilitarism and civil war in Haiti: the view from Port-au-Prince, 1994-2004. Anthropologica 2006;48:117-39.

102 Baird A. Dancing with danger: ethnographic safety, male bravado and gang research in Colombia. Qualitative Research 2018;18:342-60.

103 Lombe M, Newransky C, Crea T, et al. From rhetoric to reality: planning and conducting collaborations for international research in the global South. Soc Work 2013;58:31-40.

104 Farsides B. Commentary 2: always be prepared: Anticipating and confronting ethical challenges in the research setting. J Empir Res Hum Res Ethics 2019;14:516-8. 